\title{
SEPARATING MARGINAL UTILITY AND PROBABILISTIC RISK AVERSION
}

\begin{abstract}
This paper is motivated by the search for one cardinal utility for decisions under risk, welfare evaluations, and other contexts. This cardinal utility should have meaning prior to risk, with risk depending on cardinal utility, not the other way around. The rank-dependent utility model can reconcile such a view on utility with the position that risk attitude consists of more than marginal utility, by providing a separate risk component: a 'probabilistic risk attitude' towards probability mixtures of lotteries, modeled through a transformation for cumulative probabilities. While this separation of risk attitude into two independent components is the characteristic feature of rankdependent utility, it had not yet been axiomatized. Doing that is the purpose of this paper. Therefore, in the second part, the paper extends Yaari's axiomatization to nonlinear utility, and provides separate axiomatizations for increasing/decreasing marginal utility and for optimistic/pessimistic probability transformations. This is generalized to interpersonal comparability. It is also shown that two elementary and often-discussed properties - quasi-convexity ('aversion') of preferences with respect to probability mixtures, and convexity ('pessimism') of the probability transformation - are equivalent.
\end{abstract}

Keywords: Rank-dependent utility, risk aversion, diminishing marginal utility, strength of preference, orderings of tradeoffs.

\section{INTRODUCTION}

In rank-dependent utility, the largest stream in nonexpected utility, risk aversion is separated into two parts: the first part is 'probabilistic' risk aversion, i.e., convexity of a (cumulative) probability transformation, and the second part is diminishing marginal utility. A complete axiomatic separation of these two parts has not hitherto been obtained in the literature. ${ }^{1}$ This paper provides such, by employing the 'derived ordering of tradeoffs' method as introduced in Wakker $(1984,1989$ a). It describes an easy way to elicit and characterize utility differences, entirely independent of probabilistic risk attitudes, and has been recently used to modify prospect theory, in Tversky and Kahneman (1992) and Wakker and Tversky (1991). (A first impression of the method can be obtained from Example 9a below.) Once marginal 
utility has been isolated, it can be filtered out, and probabilistic risk attitudes can also be characterized, independently of marginal utility.

The paper is organized as follows. Section 2 describes the history of utility and the discussion whether utilities, to be used in decisions under risk, should model risk attitude, strength of preference, or both or none. The section aims to show how rank-dependent utility $(R D U)$ can provide a new answer to this classical economic question. Section 3 presents the basic general results of RDU, with Subsection 3.2 introducing the derived tradeoffs idea in decisions under risk and Subsection 3.4 giving the generalization of Yaari's (1987a) result to nonlinear utility. This provides the most general characterization of RDU presently available.

Section 4 takes up the axiomatic separation of marginal utility and probabilistic risk attitudes. Subsection 4.1 shows how to compare, under RDU, utility functions of different decisions makers, independently of their probabilistic risk attitudes. This generalizes the work of Pratt and Arrow. As a corollary, decreasing (increasing, linear) marginal utility is characterized. Subsection 4.2 shows how to compare, under RDU, the probability transformations of different decision makers, independently of their possibly different marginal utilities. The probability transformations are now interpreted as indicators of a probabilistic risk attitude. As a corollary, convex probability transformations are characterized.

The latter can also be characterized by an attractive alternative condition: quasi-convexity with respect to probability mixtures; this clearly reflects aversion to probability mixtures, i.e., probabilistic risk aversion. This characterization is presented in Subsection 4.3. While elementarily stated, its proof is complicated. To be precise, deriving quasi-convexity of preferences from convexity of the probability transformation is elementary; this was already observed by Chew (1989). The reversed implication, however, is not proved elementarily. I know from experience that specialists in the field, when first seeing this basic result, think it must have been known before, so let me emphasize that the result is new. Neither its restriction to linear utility (and/or a continuous probability transformation), nor any other close result, is available in Yaari $(1987 \mathrm{a}, \mathrm{b})$ or any other paper.

Section 5 concludes and gathers results in the main Theorem 25. 
This may be the most convenient presentation of all results of the paper except Theorems 14 and 20. Above Theorem 25, reference is given to the definitions that are used. Thus the theorem is directly accessible. Appendix 1 gives proofs, Appendix 2 the generalization to general, possibly nonquantified, outcome sets.

\section{A DISCUSSION OF UTILITY AND RISK ATTITUDE}

In traditional expected utility $(E U)$, risk attitudes are entirely modeled through the utility function. Objection has often been raised: Utility should describe an intrinsic appreciation of money, prior to probabilities or risk, and risk attitudes should consist of more than just appreciation of money. This is the point of view defended in this section.

Below, some of the history is described concerning the interpretation of utility. Next I shall argue for an intrinsic meaning of utility, which is to be relevant for risky as well as riskless applications. Then two approaches - the value-utility-difference approach and the transforming-single-outcome-probabilities approach - are described that are in line with this interpretation of utility; they incorporate components of risk attitudes separate from riskless utility. Disadvantages are mentioned. RDU is a recent and promising alternative approach to serve the same purpose. Finally, the section returns to the historical discussion of utility and argues for an interpretation of von Neumann and Morgenstern's (1944) writing on utility that differs somewhat from the prevailing interpretation.

In the early writings on EU, the difference between risky and riskless utility was not yet an issue. Bernoulli (1738) and Cramer (1728), the origins of EU, implicitly ascribed an intrinsic meaning to utility. See also Jevons (1911) and Marshall (1948, pp. 398-400). Ramsey (1931) explicitly gave at riskless interpretation to risky utility ${ }^{2}$.

The ordinalists at the beginning of this century, however, questioned the meaningfulness of (riskless) cardinal utility. While their arguments were not primarily directed towards risky utilities, they did deprive the riskless interpretation of risky utility of its basis. The remarkable utility result of von Neumann and Morgenstern (1944) did not change this 
state of affairs. Arrow (1951, p. 425) wrote, about risky utility: "... the utilities assigned are not in any sense to be interpreted as some intrinsic amount of good in the outcome...", and, about cardinal utility under certainty: "which is a meaningless concept anyway". Savage (1954, beginning of Section 5.6) wrote: ". . the now almost obsolete economic notion of utility in riskless situations, a notion still sometimes confused with the one under discussion. " 3 Luce and Raiffa (1957, p. 32) presented the viewpoint that a risky utility represents strength of preference as 'Fallacy 3'. The above citations express the representational viewpoint, which holds that utility, elicited from decisions under risk, has been proved to be applicable there, and only there. Applications to other contexts, and interpretations, are based on mere speculation; they are not based on observable preferences. This has been the dominant viewpoint since the fifties.

The idea of cardinal riskless utility has been kept alive, in spite of the ordinalists' arguments. Cardinal riskless utility is almost indispensable in many applications, such as welfare evaluations (see Fisher, 1927), the scheduling of income tax progression, dynamic decision problems, etc. For the context of risk, some references that explicitly identify risky and riskless utility are Harsanyi (1955), von Winterfeldt and Edwards (1986, p. 211 ff.), and Lopes (1987). Allais (1953) may have been the first to explicitly argue that a risk-utility should have meaning independent of risk. Indeed, the persistence of the 'mistaken idea' that concave risky utility does reflect decreasing marginal utility suggests that the idea is not entirely void of sense after all.

Under the strict representational viewpoint, risky utilities are not of great use. They are then no more than a convenient tool for modeling risk behavior, and for deriving risky decisions from other, possibly simpler, risky decisions. It is illustrative to make an, admittedly speculative, comparison with the role of mass in physics. Only when it was discovered that different ways of measurement were possible in different contexts, always leading to the same notion of mass, could mass become central in the physical sciences. Similarly for utility to become central in the social sciences, it must be one notion that can be measured in different ways in different contexts. Achieving that is worth a high price. It is hoped that the freedom that science has in modeling reality will be used to the maximum to obtain one unified 
notion of utility. This can again be compared to the physical sciences. Potential energy was introduced there for no other reason than to preserve the law of conservation of energy ${ }^{4}$; this law is worth the price of the artificial concept of potential energy.

A similar opinion on utility is found in Birnbaum and Sutton's (1992) paper on configural weight theory, a predecessor of RDU. The following is a citation from their introduction:

The principle of scale convergence states that when considering rival theories proposed to describe different empirical phenomena involving the same theoretical constructs, preference should be given to coherent theoretical systems (in which the same measurement scales can be used to account for a variety of empirical phenomena) as opposed to theoretical systems that require different measurements for each new situation . . . Configural weighting theory has the hope of resolving the inconsistent scales for utility and value measurement by separating the scaling of stimuli from the scaling of uncertainty and risk.

The section up till now has argued for an intrinsic meaning of utility, prior to risk, and applicable to many contexts. Then, if risk attitude consists of more than marginal utility, a theory must be developed that incorporates more components of risk attitude.

One such theory became popular in the 'seventies and eighties'. The idea is to model riskless utility by a cardinal 'value function', and to use an increasing nonlinear transform to carry riskless value into risky utility. See for instance Krelle (1968), Bernard (1974), Dyer and Sarin (1982), Barron, von Winterfeldt, and Fischer (1984), Farquhar and Keller (1989, Section 2.3). This approach agrees with the views defended in this section on the existence of cardinal utility ('value') prior to risk, and also allows for more components to risk attitudes than marginal (riskless) utility. However, it does not seek for one unified cardinal utility, but explicitly distinguishes between riskless and risky utility.

The value-utility-difference theory has nowadays lost popularity for the following three reasons. First, recent empirical research suggests that differences in measurements, originally attributed to a fundamental difference between value and risky utility, can be explained by fluctuations in preferences, differences between various elicitation procedures, measurement errors, and, the topic of RDU, distortions of probabilities. ${ }^{5}$ Second, the theory remains within the realm of EU, so 
is susceptible to all the paradoxes against EU. Third and most fundamentally, observability of the value function is questionable.

Another theory to incorporate more components for risk attitude than marginal utility had been popular in the psychological literature in the fifties. This approach transformed single-outcome probabilities. Thus it adds a 'probability component' to risk attitudes, which seems more natural than an additional transformation for outcomes; it does allow for one unified utility. The earliest reference is Preston and Baratta (1948). See also Edwards (1962) and several other references. The approach regained popularity when it was adopted, for a subclass of lotteries, in prospect theory by Kahneman and Tversky (1979). Earlier, the presentation in Handa (1977) had given rise to much criticism. The problem is that the transforming of single-outcome probabilities leads to a violation of stochastic dominance as soon as lotteries with more than two outcomes are involved. The first published statement of that violation is Fishburn (1978). It was discovered independently by Kahneman and Tversky (1979, p. 283-284).

Quiggin also observed that Handa's approach violates stochastic dominance. This observation, together with a general form presented by Allais (see, e.g., Allais, 1953, Section 41, Form IV), led Quiggin to the idea of RDU: One should not transform single-outcome probabilities, but cumulative probabilities, i.e., probabilities for receiving a fixed outcome or anything better (or worse, which leads to identical theories).

RDU seems to be the most popular generalization of EU nowadays. As with all deviations from EU, it allows for the modeling of the well known paradoxes, such as the Allais paradox and the Ellsberg paradox. ${ }^{6}$ It underlies the new version of prospect theory (see Tversky and Kahneman, 1992; Wakker and Tversky, 1991) that does not violate stochastic dominance, as well as the generalization of EU in Luce (1988) and Luce and Fishburn (1991). The popularity of RDU is probably explained because it is the first well-developed and axiomatized theory to permit a separate attitude towards marginal utility and probabilistic risk. It serves the purpose of this section by allowing for one unified utility.

I also want to mention what I consider weaknesses of RDU. The major weakness, in my opinion, is that a change of an outcome does 
not affect the decision weights as long as the change of outcome did not affect its ranking as compared to other outcomes, but the change of outcome may suddenly and drastically change decision weights at the moment when it does affect the ranking of outcomes. I think this sudden change of decision weights is ad hoc and can never pretend to be more than an approximation of natural processes or of a perfect normative approach. This seems to be the major weakness of RDU. A similar criticism can be formulated for the generalization of Segal (1989/1993), Green and Jullien (1988), and Chew and Wakker (1991). The non-Fréchet differentiability of RDU, as observed in Chew, Karni, and Safra (1987) and illustrated in Wakker (1989b), is a symptom of the weakness described.

Another point of concern is that no very convincing empirical support for RDU has yet been obtained. The findings in Camerer (1989) are not conclusive. Cohen and Jaffray (1988) and Tversky and Kahneman (1992) find nonlinearity in probability mainly due to the certainty effect, i.e. if probabilities 0 or 1 are involved. The latter suggests that RDU is too general. Munier and Abdellaoui (1991), on the contrary, find nonlinearities throughout the domain.

I have deferred until the end the discussion of the subtle viewpoints of von Neumann and Morgenstern (1944) (vNM) on risky versus riskless utility. Fishburn (1989) gave an informative exposition, upon which the analysis here and above has drawn heavily. In several places, e.g. at the end of Section 3.2 and in footnote 1 there, vNM write that their risky utility makes it possible to order utility differences. This has led many (e.g. Allais, 1979, p. 592) to claim that vNM interpreted their risky utility as identical to riskless cardinal utility. However, vNM write in the footnote on p. 630-631, about their utility difference terminology (italics added here): "merely to facilitate the verbal discussion - they are not part of our rigorous, axiomatic, system." The ordering of utility differences concerns risky utility differences. There is no formal reason to identify them with some notion of riskless utility difference. This 'retraction' of vNM, as well as other similar retractions about orderings of utility differences, has been emphasized by Fishburn (1989).

On the basis of these writings of vNM, the representational viewpoint has been ascribed to them. But maybe their position is in 
between. They only say that no riskless formalization of their utility has been given. This does not exclude a future possibility, and desirability, to do so. At several places (e.g. the beginning of 3.2.1), vNM point out that one should be careful to exclude future developments of theories. Several parts of their text suggest to me that they have in mind one notion of utility for the entire economic science. The very motivation for their analysis has been to obtain a cardinal utility that is relevant for another context: game theory. The same cardinal utility that provides an expectation representing individuals' preferences over randomized outcomes, is also used to provide the unit of exchange between players. I think the applicability of risky utility as means of exchange between players is as questionable as its applicability to welfare theory, or any other case of decisions under certainty.

This section has defended a unified notion of utility that is prior to, but relevant for, risk. RDU can reconcile this with the position that there is more to risk attitudes than marginal utility, by adding a probabilistic risk attitude component. I hope that the reader will at least consider the described position as tenable. The rest of the paper gives axiomatic results for RDU that can be read and used independently of the viewpoints of this section. The methods and results have, however, been motivated by the opinions expressed above. The adopted tool of derived orderings of tradeoffs appeals to cardinal utility, independent of probabilistic risk attitudes.

\section{RANK-DEPENDENT UTILITY FOR DECISIONS UNDER RISK}

\subsection{Elementary Definitions}

$\mathscr{P}$ denotes the set of probability distributions, called lotteries, over an interval $[0, M]$, with $M$ an arbitrary fixed positive number; elements of $[0, M]$ are called outcomes. By $\geq$ we denote the preference relation of a decision maker on $\mathscr{P}$, with $\sim,>, \leq$, and $<$ as usual; $\geq$ is a weak order if it is complete $(P \geq Q$ or $Q \geq P$ for all $P, Q)$ and transitive. A function $V: \mathscr{P} \rightarrow \mathbb{R}$ represents $\geq$ if $[P \geq Q] \Leftrightarrow[V(P) \geqslant V(Q)]$. (The term utility will be used for the function $U$ below; generalized expectations of $U$ will represent $\geq$.) Cardinal is in this paper no more than an 
abbreviation of the intractable 'unique up to a positive affine transformation'.

In descriptive contexts it is well known that preferences depend on the way lotteries are presented to subjects. This paper does not address that question, and assumes that preferences depend only on probability distributions and not on the way of presentation.

DEFINITION 1. Rank-dependent utility (RDU) holds if there exist a strictly increasing continuous utility function $U:[0, M] \rightarrow \mathbb{R}$ and a strictly increasing probability transformation $\varphi:[0,1] \rightarrow[0,1]$ with $\varphi(0)=0$ and $\varphi(1)=1$, such that $\geq$ is represented by

$$
P \mapsto \int_{\mathbb{R}_{+}} \varphi \circ G_{U, P} \mathrm{~d} \tau+\int_{\mathbb{R}_{-}}\left[\varphi \circ G_{U, P}-1\right] \mathrm{d} \tau
$$

Here $G_{U, P}$ is the decumulative distribution function of $U$ under $P$, i.e. $G_{U, P}: \tau \mapsto P(\{\alpha \in[0, M]: U(\alpha) \geqslant \tau\})$. The integral is the rank-dependent utility (RDU) of the distribution $P$.

Continuity of $\varphi$ has not been incorporated in the definition of RDU, because there is interest in discontinuities, primarily at 0 and 1 . The characterization in Theorem 12 leaves continuity of $\varphi$ optional. $\mathscr{P}^{s}$ denotes the set of simple lotteries, i.e., probability distributions that assign probability one to a finite set. A simple lottery is denoted by $\left(p_{1}, x_{1} ; \ldots ; p_{n}, x_{n}\right)$, where $x_{1} \geqslant \cdots \geqslant x_{n}$ and $p_{j}>0$ for all $j$. Obviously, this designates the probability distribution that assigns probability $p_{1}$ to outcome $x_{1}$, etc. Note that the outcomes are rank-ordered. Axioms will be formulated by means of this notation in such a way that the notation is relevant. For illustration, a small deviation of this notation occurs in Example 9c; it will illustrate the crucial role of rank-ordering in RDU.

Misunderstandings have arising in the literature as a consequence of inaccurate notation. Hence it is emphasized that subsequent $x_{j}$ s are allowed to be identical. For example, $\left(p_{1}, \alpha ; p_{2}, \alpha ; p_{3}, x_{3} ; \ldots ; p_{n}\right.$, $\left.x_{n}\right)$ is identical to $\left(p_{1}+p_{2}, \alpha ; p_{3}, x_{3} ; \ldots ; p_{n}, x_{n}\right)$. This identity is not an assumption, but a logical necessity, these notations merely being 
two different ways of writing the same probability distribution. For a simple lottery $\left(p_{1}, x_{1} ; \ldots ; p_{n}, x_{n}\right)$, RDU can be written as a sum:

$$
\begin{aligned}
& R D U\left(p_{1}, x_{1} ; \ldots ; p_{n}, x_{n}\right)=\sum_{i=1}^{n} \lambda_{i} U\left(x_{i}\right), \text { with } \\
& \lambda_{i}:=\varphi\left(p_{1}+\cdots+p_{i}\right)-\varphi\left(p_{1}+\cdots+p_{i-1}\right) .
\end{aligned}
$$

In the above formula, $\lambda_{1}=\varphi\left(p_{1}\right)$. The decision weights $\lambda_{i}$ are a kind of marginal $\varphi$ contribution to cumulative probabilities; they sum to one. One verifies elementarily that different notations for one same probability distribution, by different ways of 'splitting-up' of probabilities of one same outcome, do not affect the RDU value in (2).

The following result will be used in Subsection 4.3. Its simple proof is given immediately because it illustrates the nature of RDU.

OBSERVATION 2. RDU is convex, concave, or linear (with respect to probability mixtures) if and only if $\varphi$ is.

Proof. We only consider convexity. The decumulative distribution function of a (probability) mixture of probability distributions being the same mixture of decumulative distribution functions, convexity of $\varphi$ implies the same for RDU by (1). Conversely, if we set $U(M)=1$, $U(0)=0$, then the equality

$$
\varphi(p)=R D U(p, M ; 1-p, 0)
$$

shows that convexity of RDU implies convexity of $\varphi$.

Throughout the paper the following assumption is used, where $P$ stochastically dominates $Q$ if $P(]-\infty, \tau]) \leqslant Q(]-\infty, \tau])$ for all real $\tau$.

\section{ASSUMPTION 3.}

(i) Weak ordering.

(ii) $[($ Strict) Stochastic Dominance $] P>Q$ whenever $P \neq Q$ and $P$ stochastically dominates $Q$. 


\subsection{Derived Tradeoffs}

This subsection adapts the idea of derived (orderings of) tradeoffs, used in several previous papers of the author in decisions under uncertainty and multiattribute utility theory (see e.g. Wakker, 1989a), to the present context of decisions under risk. I hope that this paper, in giving the most general characterization of RDU presently available, and in being the first to obtain a complete axiomatic separation of marginal utility and probabilistic risk attitudes, will increase the interest in derived tradeoffs. Recently, Tversky and Kahneman (1992) and Wakker and Tversky (1991) used derived tradeoffs to obtain a new version of prospect theory that avoids problems concerning violation of stochastic dominance/transitivity; Wakker and Tversky (1991) give further motivations for the use of derived tradeoffs.

The formal results of this subsection are given in the displayed and numbered definitions, formulas, and lemmas. The rest of the subsection elucidates. Examples are given at the end of the subsection.

The notation with star superscripts below indicates that the relation is quarternary; it might be interpreted as a revealed ordering of (a kind of) strengths of preferences. The notation $\left(p_{1}, x_{1} ; \ldots ; p_{i}, \alpha ; \ldots ; p_{n}\right.$, $\left.x_{n}\right)$ is short for $\left(p_{1}, x_{1} ; \ldots ; p_{i-1}, x_{i-1} ; p_{i}, \alpha ; p_{i+1}, x_{i+1} ; \ldots ; p_{n}, x_{n}\right)$.

DEFINITION 4. For outcomes $\alpha, \beta, \gamma, \delta$, we write $[\alpha ; \beta] \geq^{*}[\gamma ; \delta]$, or $\alpha \beta \geq^{*} \gamma \delta$ for short, if

$$
\begin{aligned}
& \left(p_{1}, x_{1} ; \ldots ; p_{i}, \alpha ; \ldots ; p_{n}, x_{n}\right) \geq\left(p_{1}, y_{1} ; \ldots ; p_{i}, \beta ; \ldots ; p_{n}, y_{n}\right) \text { and } \\
& \left(p_{1}, x_{1} ; \ldots ; p_{i}, \gamma ; \ldots ; p_{n}, x_{n}\right) \leq\left(p_{1}, y_{1} ; \ldots ; p_{i}, \delta ; \ldots ; p_{n}, y_{n}\right)
\end{aligned}
$$

for some $i, p_{1}, \ldots, y_{n}$.

Note that the probabilities $p_{1}, \ldots, p_{n}$ were kept fixed throughout, and that our conventions of notation implicitly imply that $x_{i-1} \geqslant \alpha \geqslant x_{i+1}$, $y_{i-1} \geqslant \beta \geqslant y_{i+1}, \quad x_{i-1} \geqslant \gamma \geqslant x_{i+1}, \quad$ and $y_{i-1} \geqslant \delta \geqslant y_{i+1}$. Substituting RDU for the preferences in Definition 4 gives $a \geqslant$ and $a \leqslant$ inequality, where, with $\lambda_{i}$ as in (3), the only difference between the two inequalities is that the terms $\lambda_{i} U(\alpha), \lambda_{i} U(\beta)$ above have been replaced 
by $\lambda_{i} U(\gamma), \lambda_{i} U(\delta)$ below. Cancelling the common positive factor $\lambda_{i}$ then shows:

$$
\begin{aligned}
& \text { If RDU holds then: } \alpha \beta \geq^{*} \gamma \delta \Rightarrow U(\alpha)-U(\beta) \\
& \quad \geqslant U(\gamma)-U(\delta) .
\end{aligned}
$$

So $\geq^{*}$ is in agreement with the ordering of utility differences and may serve as a tool for the actual elicitation of utility. The reversed implication also holds under certain conditions, such as continuity of the probability transformation and continuity of utility. This can be derived from Theorem 12 .

DEFINITION 5. We write $\alpha \beta>^{*} \gamma \delta$ if in Definition 4 we have $<$ instead of $\leq$; we write $\alpha \beta \sim{ }^{*} \gamma \delta$ if in Definition 4 we have $\sim$ instead of $\leq$ and $\geq$.

The following is similar to (4).

If RDU holds then:

$$
\alpha \beta \sim{ }^{*} \gamma \delta \Rightarrow U(\alpha)-U(\beta)=U(\gamma)-U(\delta)
$$

and

$$
\alpha \beta>^{*} \gamma \delta \Rightarrow U(\alpha)-U(\beta)>U(\gamma)-U(\delta) .
$$

For verbal elucidation of the $*$ relations above, consider $>^{*}$, with $<$ instead of $\leq$ in Definition 4. Replacement of $\alpha, \beta$ by $\gamma, \delta$ has caused a reversal of preference. The probabilities and the rank-ordering of outcomes have been kept fixed, so that also the probabilistic risk effects $^{7}$ have been kept constant for all preferences. The change of preference, being observed under constant probabilistic risk, must be explained by the change in outcomes: the 'tradeoff' $\alpha \beta$ (receiving $\alpha$ instead of $\beta$ ) must be a stronger improvement (less serious loss) than the tradeoff $\gamma \delta$. This is reflected in Equation (6). Examples which further illustrate the intuition of the condition are given in Wakker and Tversky (1991).

Next we introduce the main condition in the characterization of RDU. In earlier papers by the author the expression 'absence of 
contradictory tradeoffs' was used for conditions like the one below. This paper follows the preferable terminology introduced in Tversky and Kahneman (1992).

DEFINITION 6 . We say that $\geq$ satisfies tradeoff consistency if there do not exist outcomes $\alpha, \beta, \gamma, \delta$ such that both $\alpha \beta \geq^{*} \gamma \delta$ and $\gamma \delta>^{*} \alpha \beta$.

An immediate consequence of (4) and (6) is:

LEMMA 7. RDU implies tradeoff consistency.

Theorem 12 shows that under usual conditions tradeoff consistency is not only necessary, but also sufficient, for RDU. To emphasize the present context of rank-ordered outcomes and to distinguish from other versions of tradeoff consistency, the terms 'rank-ordered tradeoff consistency' or 'comonotonic tradeoff consistency' would be more appropriate. The above version of tradeoff consistency being the only one in this paper, qualifying adjectives have been omitted, and no indexes have been added in the notation to indicate comonotonicity in the $*$ relations $\geq^{*},>^{*}, \sim^{*}$. Comonotonicity has been introduced in Schmeidler (1989, first version 1982) and has also been used in Yaari (1987a, first version 1984).

It may be argued that the use of $*$ relations in characterizations violates a 'rule of the game' of representation theory. Representation theorems give characterizations, i.e., necessary and sufficient conditions for preferences, for the applicability of quantitative models such as RDU. The characterizations, given for instance in Statement (ii) in the representation Theorem 25 below, should 'translate' the meaning of the theoretical Statement (i) (claiming applicability of RDU) into the 'empirical language' directly in terms of the observable primitives, being the preferences, outcomes and associated probabilities. Therefore the $*$ relations, being derived rather than primitive, should not be used in characterizations, it seems. However, the $*$ relations are derived from the preferences in a very direct manner, so that it is easy to see the empirical content of statements in terms of these relations. It 
is easy to replace the * relations by their definitions, leading to conditions directly in terms of preferences and directly testable; see Observation 8.

I think that the formulations in terms of the * conditions are transparent, and that this outweighs the disadvantage of being one step further away from empirical reality. This does not hold, for example, for conditions directly in terms of utilities. In general it is not easy and involves many steps to derive utilities from preferences, thus to test conditions for utilities. So conditions for utilities should not be used in the characterizing empirical statements in representation theory.

The observation below presents the reformulation of tradeoff consistency directly in terms of conditions for preferences.

OBSERVATION 8. Tradeoff consistency is satisfied if and only if

$$
\begin{aligned}
& \left(p_{1}, x_{1} ; \ldots ; p_{i}, \alpha ; \ldots ; p_{n}, x_{n}\right) \geq\left(p_{1}, y_{1} ; \ldots ; p_{i}, \beta ; \ldots ; p_{n}, y_{n}\right) \\
& \left(p_{1}, x_{1} ; \ldots ; p_{i}, \gamma ; \ldots ; p_{n}, x_{n}\right) \leq\left(p_{1}, y_{1} ; \ldots ; p_{i}, \delta ; \ldots ; p_{n}, y_{n}\right)
\end{aligned}
$$

and

$$
\begin{aligned}
& \left(q_{1}, v_{1} ; \ldots ; q_{j}, \gamma ; \ldots ; q_{m}, v_{m}\right) \geq\left(q_{1}, w_{1} ; \ldots ; q_{j}, \delta ; \ldots ; q_{m}, w_{m}\right) \\
& \left(q_{1}, v_{1} ; \ldots ; q_{j}, \alpha ; \ldots ; q_{m}, v_{m}\right)<\left(q_{1}, w_{1} ; \ldots ; q_{j}, \beta ; \ldots ; q_{m}, w_{m}\right)
\end{aligned}
$$

for no $i, p_{1}, \ldots, w_{m}$.

Finally, some examples are given for the above conditions.

Example $9 a$ (Violation of tradeoff consistency). Preferences

$$
\begin{aligned}
& \left(\frac{3}{8}, 60 ; \frac{2}{8}, 50 ; \frac{3}{8}, 31\right) \geq\left(\frac{3}{8}, 61 ; \frac{2}{8}, 51 ; \frac{3}{8}, 30\right) \text { and } \\
& \left(\frac{3}{8}, 60 ; \frac{2}{8}, 50 ; \frac{3}{8}, 41\right) \leq\left(\frac{3}{8}, 61 ; \frac{2}{8}, 51 ; \frac{3}{8}, 40\right)
\end{aligned}
$$

reveal $[31 ; 30] \geq *[41 ; 40]$. Substituting $\operatorname{RDU}$ gives $U(31)-U(30) \geqslant$ $U(41)-U(40)$, in accordance with Formula (4): an ordering of utility 
differences has been revealed. If the following preferences would also be observed:

$$
\begin{aligned}
& \left(\frac{1}{2}, 41 ; \frac{1}{4}, 20 ; \frac{1}{4}, 10\right) \geq\left(\frac{1}{2}, 40 ; \frac{1}{4}, 21 ; \frac{1}{4}, 11\right) \text { and } \\
& \left(\frac{1}{2}, 31 ; \frac{1}{4}, 20 ; \frac{1}{4}, 10\right)<\left(\frac{1}{2}, 30 ; \frac{1}{4}, 21 ; \frac{1}{4}, 11\right),
\end{aligned}
$$

then these would reveal $[41 ; 40]>*[31 ; 30]$. Together with the revealed ordering of tradeoffs found above this gives a violation of tradeoff consistency. Indeed, under RDU the result would be $U(41)-U(40)>$ $U(31)-U(30)$, contradicting the ordering of utility differences found above. The four observed preferences falsify RDU.

Example $9 b$ (Comonotonic sure-thing principle). The comonotonic sure-thing principle is violated if and only if there exist preferences of the kind

$$
\begin{aligned}
& \left(p_{1}, x_{1} ; \ldots ; p_{i}, \alpha ; \ldots ; p_{n}, x_{n}\right) \geq\left(p_{1}, y_{1} ; \ldots ; p_{i}, \alpha ; \ldots ; p_{n}, y_{n}\right) \\
& \left(p_{1}, x_{1} ; \ldots ; p_{i}, \gamma ; \ldots ; p_{n}, x_{n}\right)<\left(p_{1}, y_{1} ; \ldots ; p_{i}, \gamma ; \ldots ; p_{n}, y_{n}\right) .
\end{aligned}
$$

That is, it is violated if preferences are not independent of common outcomes. This condition is weaker than the expected-utility sure-thing principle because it is only imposed on lotteries with rank-ordered outcomes. Preferences as above reveal the peculiar $[\alpha ; \alpha]>^{*}[\gamma ; \gamma]$, which by (6) immediately falsifies $\mathrm{RDU}$. By $[\gamma ; \gamma] \geq \geq^{*}[\alpha ; \alpha]$, as resulting from reflexivity and the trivial

$$
\begin{aligned}
& \left(p_{1}, x_{1} ; \ldots ; p_{i}, \gamma ; \ldots ; p_{n}, x_{n}\right) \geq\left(p_{1}, x_{1} ; \ldots ; p_{i}, \gamma ; \ldots ; p_{n}, x_{n}\right) \\
& \left(p_{1}, x_{1} ; \ldots ; p_{i}, \alpha ; \ldots ; p_{n}, x_{n}\right) \leq\left(p_{1}, x_{1} ; \ldots ; p_{i}, \alpha ; \ldots ; p_{n}, x_{n}\right),
\end{aligned}
$$

a formal violation of tradeoff consistency is obtained.

Example $9 c$ (The Allais Paradox). Many authors have shown that the Allais paradox can be described by RDU. The example is nevertheless presented here because it illustrates the notations and conditions of this paper. They will slightly deviate here from the main body of the 
paper, as will be explained shortly. Suppose that we observe, with $M$ denoting $\$ 1000000$,

$$
\begin{aligned}
& \left(\frac{1}{10}, M ; \frac{89}{100}, M ; \frac{1}{100}, M\right) \geq\left(\frac{1}{10}, 5 M ; \frac{89}{100}, M ; \frac{1}{100}, 0\right) \text { and } \\
& \left(\frac{1}{10}, M ; \frac{89}{100}, 0 ; \frac{1}{100}, M\right)<\left(\frac{1}{10}, 5 M ; \frac{89}{100}, 0 ; \frac{1}{100}, 0\right) .
\end{aligned}
$$

It may seem that, by Definition 4 (with $i=2, p_{i}=0.89$ ), $[M ; M]$ $>^{*}[0 ; 0]$, which by (6) immediately leads to a falsification of RDU. Together with $[0 ; 0] \geq *[M ; M]$, as trivially implied by $\left(\frac{1}{2}, M ; \frac{1}{2}, 0\right) \geq\left(\frac{1}{2}\right.$, $\left.M ; \frac{1}{2}, 0\right)$ and $\left(\frac{1}{2}, M ; \frac{1}{2}, M\right) \leq\left(\frac{1}{2}, M ; \frac{1}{2}, M\right)$ (Definition 4 with $i=2$ ), this would also violate tradeoff consistency. Indeed, usual additive EU can be falsified by similar reasonings. Still, RDU is not falsified because it is crucial for $R D U$ that the outcomes are rank-ordered. In the notation $\left(\frac{1}{10}, M ; \frac{89}{100}, 0 ; \frac{1}{100}, M\right)$ for the third lottery in the above preferences, the outcomes were not rank-ordered. Once the outcomes are rank-ordered, the violation of tradeoff consistency disappears. Indeed, RDU can model the Allais preferences, as is well known, e.g. by taking utility $=$ identity and $\varphi: p \mapsto p / 2$ for all $p \leqslant 0.99$.

\subsection{Further Conditions}

This subsection gives some more conditions of a technical nature, used in the main theorem. This paper follows the usual terminology, where probability measures are countably additive.

One continuity condition is assumed in the main results. It only considers simple lotteries with fixed probabilities and variations in outcomes and is weaker, so less restrictive, than most continuity conditions used in the literature; these are further discussed at the end of Appendix 2. By $[0, M]_{\downarrow}^{n}$ we denote the set of rank-ordered $n$-tuples from $[0, M]$, i.e.,

$$
[0, M]_{\downarrow}^{n}:=\left\{\left(x_{1}, \ldots, x_{n}\right) \in[0, M]^{n}: x_{1} \geqslant \cdots \geqslant x_{n}\right\} .
$$

DEFINITION 10. The preference relation $\geq$ satisfies simple-continuity if, for any fixed probability vector $\left(p_{1}, \ldots, p_{n}\right)$, the preference relation induced on $[0, M]_{\downarrow}^{n}$ is continuous. 
Instead of continuity on nonsimple lotteries, this paper uses the certainty-equivalent condition, requiring existence for every probability distribution $P$ of a certainty equivalent $\mathrm{CE}(P) \in[0, M]$, such that $\mathrm{CE}(P) \sim P$. Again, it is weaker than the infinite-dimensional continuity conditions as used in the literature.

The following conditions characterize continuity of the strictly increasing probability transformation. Because there is empirical interest in discontinuity at 0 and 1 plus continuity on $] 0,1$ [, we separate these continuities and leave each combination of them optional in Theorem 12.

DEFINITION 11 . We say that $\geq$ satisfies continuity in probabilities on ]0,1[ if, for all probabilities $p$ :

$$
\begin{aligned}
& \text { If }(p, M ; 1-p, 0)>(1, \alpha) \text { then there exists } q<p \text { such that } \\
& \text { still }(q, M ; 1-q, 0)>(1, \alpha),
\end{aligned}
$$

and the same holds if the two preferences and the inequality are reversed. We say that $\geq$ satisfies continuity in probabilities at 1 if (8) holds for $p=1 ; \geq$ satisfies continuity in probabilities at 0 if (8) holds for $p=0$ with reversed preferences and inequality. ${ }^{8}$

The following solvability condition for the increasing probability transformation, similar to Quiggin (1982, Axiom 3), can be seen to be equivalent to continuity at all probabilities: for every outcome $\beta$ there exists a probability $p$ such that $(1, \beta) \sim(p, M ; 1-p, 0)$.

\subsection{Extending Yaari's (1987a) Representation to Nonlinear Utility}

THEOREM 12. Let $\geq$ be a preference relation on $\mathscr{P}$. RDU holds if and only if $\geq$ is a weak order that satisfies the certainty-equivalent condition, simple-continuity, stochastic dominance, and tradeoff consistency. The probability transformation is uniquely determined, utility is cardinal. Continuity conditions of the probability transformation agree with the related continuity conditions of $\geq$ in probability. 
This theorem gives the most general characterization of RDU, presently available. Continuity of $\varphi$ at 0,1 , and on ]0,1[ is optional, and the result can without any problem be adapted to contexts where not all probability distributions are given. Any fixed subset of $[0,1]$ will do as set of available probabilities; the proof needs no adaptations for this. Further, the approach of this paper can easily be extended to general intervals, nonincreasing utility, to multidimensional outcomes, and even nonquantitative outcomes; Appendix 2 describes the extension to any connected topological space. The remainder of this subsection discusses related literature.

As compared to Quiggin (1982), the restriction $\varphi\left(\frac{1}{2}\right)=\frac{1}{2}$ has been deleted, discontinuity of $\varphi$ is allowed for, and nonsimple probability distributions have been included; Quiggin's analysis also uses continuity in outcomes in an implicit manner. Elucidations to the mathematics in Quiggin (1982) are provided in Quiggin and Wakker (1992).

As compared to Yaari (1987a), the restrictions of linearity of utility and continuity of $\varphi$ have been deleted. Linearity of utility was also relaxed in Segal (1989), a result that was used in Segal (1990). The result was criticized in Wakker (1993b), and corrected in Segal (1993). His characterizing condition employs epigraphs of distribution functions and is not intuitive in terms of preferences. It applies to onedimensional monetary outcomes and finite-dimensional commodity bundles, and implies continuity of utility and of the probability transformation. Luce (1988), Segal (1990), and Luce and Fishburn (1991) use a two-stage approach to obtain rank-dependent forms.

Theorem 1 in Chew (1989) is the result in the literature that is most similar to Theorem 12. Chew formulates his characterization for functionals, but in the first sentence of Section 8 indicates the way to reformulate his results for preference theory (through certainty equivalents). Instead of the requirement of tradeoff consistency, Chew uses a 'Weak Commutativity' condition, which can be seen to be a version of the bisymmetry condition from functional equations, as introduced in decision theory by Pfanzagl (1968) and also studied in Section 6.9 of Krantz et al. (1971). Chew uses ('compact') continuity with respect to weak convergence; again, this requires both continuity in outcomes and in probabilities, and leads not only to continuity of utility but also 
to continuity of the transformation $\varphi$. Also Chew's weak commutativity axiom uses essentially the availability of certainty equivalents (implied by his continuity), so that his intuitive axiom involves structural/technical assumptions.

Also Chew and Epstein (1989) provided a characterization of RDU, for monetary outcomes and continuity of utility and probability transformation. Corrections for their work were supplemented in Chew, Epstein and Wakker (1993). Chateauneuf (1990) proposed two axioms, A.4 and A.5, which together are also necessary and sufficient for RDU for simple distributions; here $\mathscr{C}$ is a connected compact metric space (see also Appendix 2). Axiom A.4 is a comonotonic sure-thing principle, A.5 is an attractive adaptation of mixture independence to RDU which, like tradeoff consistency, implies proportionality of the additive value functions. Continuity of $\varphi$ is again imposed. A first version of our Theorem 12, with an underlying state space made explicit, appeared in Wakker (1987, Observation 7.6.1 plus Theorem 6.8).

Nakamura (1992, Proposition 2) also makes explicit the underlying [0,1] state space. He considers only simple probability distributions and uses the 'algebraic approach' initiated by Krantz et al. (1971). Wakker (1988) argues for the preferability of this approach over the topological approach; the latter, however, is more customary and hence is used in this paper (see Appendix 2 for full generality). Nakamura's nontechnical axiom is a generalization of bisymmetry axioms to multisymmetric structures and is similar to Chew's (1989) weak commutativity. As Chew's axiom, it needs availability of certainty equivalents in its formulation. For uncertainty, an appealing variation on the multisymmetric axiom was introduced in Gul (1992) for additive EU and has been used in Chew and Karni (1991) for rank-dependent theories; Wakker (1991b, Theorem 8) adapted Theorem 12 to uncertainty and to the algebraic approach; his structural assumptions are somewhat less restrictive than the ones used in the above works and, for instance, do not imply the existence of certainty equivalents. All works discussed so far for the context of uncertainty assumed finite state spaces. Extensions to infinite state spaces have been given in Wakker (1993c). 


\section{DISENTANGLING PROBABILISTIC RISK ATTITUDES AND} MARGINAL UTILITY

Before turning to our results in Subsection 4.1, we discuss some literature. Axioms for the distinction between attitudes towards probabilistic risk and attitudes toward wealth have not yet been provided in the literature. Chew, Karni, and Safra (1987) use the condition that a mean-preserving increase in risk is never favorable, to characterize the joint concavity of utility and convexity ${ }^{9}$ of $\varphi$.

That same condition is used in Yaari (1987a, see also Yaari, 1987b, p. 176) as a necessary and sufficient condition for convexity of $\varphi$. Yaari does not need to deal with the separation of marginal utility and probabilistic risk attitudes because other conditions in his approach already imply constant marginal utility. Also Chateauneuf (1988) characterizes convexity of $\varphi$, mainly by 'uncertainty aversion' axioms. He does not need to reckon with marginal utility because, as in Yaari (1987a), the axioms imply constant marginal utility. Chateauneuf indicates the desirability of the generalization to nonlinear utility in Section 5; the present paper provides that generalization. See also Quiggin (1982, p. 328, bottom) and Yaari (1987a, p. $961.11-12$ and p. $1101.3-6)$. Wakker (1990b, Corollary 9) characterizes convexity of $\varphi$ in a different way, directly in terms of comonotonicity, by requiring the usual independence implication for mixtures with respect to outcomes to hold whenever only the lotteries in the dispreferred mixture are comonotonic, and the other lotteries can be general. Again, the result, like Yaari's and Chateauneuf's, crucially depends on the implied linearity of utility.

Hilton (1988) considers risk premiums, and finds a way to separate these into a term related to the transformation $\varphi$ of probabilities and a term similar to the traditional Pratt/Arrow measure. Hilton's separation, however, requires knowledge of $U$ and $\varphi$, so is theoretical, and does not provide a separated behavioral axiomatization directly in terms of testable properties of preferences. Chateauneuf and Cohen (1990) study the definition of risk aversion that requires preference of expected values over lotteries; this is weaker than aversion to meanpreserving risk. They show that neither diminishing marginal utility, 
nor convexity of the probability transformation, is implied. An alternative condition to characterize convexity of the probability transformation for general utility, and different from the conditions of this paper, was obtained independently by Wakker (1986, Section VI.11), Segal (1987), and Chateauneuf (1990).

\subsection{Decreasing/Increasing Marginal Utility, and the Pratt/Arrow} Comparisons of Marginal Utility, Generalized to RDU

This subsection generalizes the classical Pratt/Arrow results concerning marginal utility from EU to RDU. We characterize the case where one decision maker's utility is a concave transform of a second decision maker's utility, i.e., the first utility shows a 'stronger decrease of marginal utility'. As a corollary, characterizations are obtained of concave, convex, and linear, utility. In the interpretation of this paper, these results reflect a stronger decrease of marginal utility where marginal utility is prior to risk attitude. The results of this subsection are based on the techniques as developed in Wakker (1989a, Chapter VII) for additive subjective expected utility.

In $U^{1}, U^{2}$, superscripts are general superscripts and do not designate exponents. The $1^{*}$ and $2^{*}$ relations below are derived from $\geq^{1}$ and $\geq^{2}$ in the usual way. The characterizing conditions given below reflect the corresponding properties of marginal utility in a transparent manner.

DEFINITION 13. We say that $\geq^{2}$ exhibits a stronger decrease of marginal utility than $\geq^{1}$ if, for all $\alpha \geqslant \beta \geqslant \gamma \geqslant \delta,\left[\alpha \beta \leq^{1 *} \gamma \delta \&\right.$ $\alpha \beta>^{2 *} \gamma \delta$ ] is excluded.

The condition describes what intuition suggests: a high-payments tradeoff $\alpha \beta$ should not come out better for the decision maker with the stronger decrease of marginal utility.

THEOREM 14. Suppose that RDU holds for $\geq^{1}, \varphi^{1}, U^{1}$ as well as $\geq^{2}, \varphi^{2}, U^{2}$. Then the following two statements are equivalent: 
(i) $U^{2}=\psi \circ U^{1}$ for a continuous, concave, and strictly increasing $\psi$ on $U^{1}([0, M])$.

(ii) $\geq^{2}$ exhibits a stronger decrease of marginal utility than $\geq^{1}$.

The proof of the implication (i) $\Rightarrow$ (ii) may clarify: suppose that $U^{1}(\alpha)-U^{1}(\beta) \leqslant U^{1}(\gamma)-U^{1}(\delta)$ and $U^{2}(\alpha)-U^{2}(\beta)>U^{2}(\gamma)-$ $U^{2}(\delta)$ for $\alpha \geqslant \beta \geqslant \gamma \geqslant \delta$, as implied by the preferences excluded in Definition 13, and by (4) and (6). Then, in terms of the $U^{1}$ scale, $U^{2}$ exhibits a strict increase in marginal utility. This precludes that $U^{2}=$ $\psi * U^{1}$ for a concave $\psi$ on $U^{1}([0, M])$. So a violation of (ii) implies a violation of (i), which establishes the implication (i) $\Rightarrow$ (ii).

Of course, in Definition 13 one can define a weaker decrease of marginal utility by reversing the preferences between the square brackets or by interchanging the role of $\geq^{1}$ and $\geq^{2}$. This characterizes a convex transformation $\psi$ in statement (i) in Theorem 14. The combination of the two conditions is called identical marginal utility and leads to utilities that are related by a linear transformation, i.e., that are identical given cardinality. As a corollary of Theorem 14, results are obtained concerning increasing and decreasing marginal utility. For clarity we write $[\alpha ; \beta]$ instead of $\alpha \beta$ for tradeoffs.

DEFINITION 15. We say that:

$\geq$ exhibits decreasing marginal utility if $[\alpha+\epsilon ; \beta+\epsilon]>{ }^{*}[\alpha ; \beta]$ is excluded for all outcomes in question with $\alpha>\beta, \epsilon>0$;

$\geq$ exhibits increasing marginal utility if $[\alpha ; \beta]>{ }^{*}[\alpha+\epsilon ; \beta+\epsilon]$ is excluded for all outcomes as above;

$\geq$ exhibits constant marginal utility if it exhibits both decreasing and increasing marginal utility.

Again, the conditions describe what intuition suggests: e.g., under decreasing marginal utility the strength of preference (interpreting utility differences like that) of $\alpha+\epsilon$ over $\beta+\epsilon$ should not exceed that of $\alpha$ over $\beta$. It is easily seen that a decision maker exhibits decreasing marginal utility if and only if he exhibits a stronger decrease of marginal utility than an expected value maximizer. Similar things hold 
for increasing/constant marginal utility. This illustrates the convenience of comparability of marginal utility, independent of attitudes towards probabilized risk. The fact that expected value preferences are linear in probabilities and general RDU preferences are not, does not pose any problem. Thus an immediate corollary of Theorem 14 is:

COROLLARY 16. Under RDU, $U$ is concave (convex, linear) if and only if $\geq$ exhibits decreasing (increasing, constant) marginal utility.

\subsection{Characterizing Probabilistic Risk Aversion Independently of Marginal Utility}

We have seen above that the $*$ relations provide the means to measure phenomena related to marginal utility, independently of attitudes towards probabilistic risk. So these marginal utility phenomena can be 'filtered out', and subsequently the effects of attitudes towards probabilistic risk can be isolated. This makes it possible to compare the attitudes towards probabilistic risk of two decision makers even if they have different marginal utility. Given the great generality of this method of comparison, it cannot be expected to be very simple; Table 1 shows that the characterizing condition actually involves a scheme of five preferences for each decision maker. Nevertheless it is hoped that the formulation of the characterizing condition in Definition 19 by means of derived tradeoffs (which underlies Definition 17) will make the idea transparent.

As an illustration, consider two 'very' elementary probability distributions $P:=(p, M ; 1-p, 0), Q:=(q, M ; 1-q, 0)$. We want to see how, for some probabilistic mixture $\lambda P+(1-\lambda) Q$, the first decision maker compares this 'increased probabilistic risk' with the 'midpointoutcome' that has as utility the midpoint of the RDU values of the lotteries, and how the second decision maker does this. We want to find out if the second decision maker has systematically higher aversion to the increased probabilistic risk than the first. This is the case if he at least as often disprefers the probabilistic mixture to his midpointoutcome as does the first decision maker. In the theory of RDU that will imply (e.g. with $U(0)=0, U(M)=1$ ) 


$$
\begin{aligned}
\varphi^{1}(\lambda p+(1-\lambda) q) \leqslant & \left(\varphi^{1}(p)+\varphi^{1}(q)\right) / 2 \Rightarrow \varphi^{2}(\lambda p \\
& +(1-\lambda) q) \leqslant\left(\varphi^{2}(p)+\varphi^{2}(q)\right) / 2 .
\end{aligned}
$$

As elaborated in the proof of Theorem 20, this condition implies under continuity that $\varphi^{2}$ is a convex transform of $\varphi^{1}$. The major problem, finding a way to detect behaviorally what the midpoint-outcomes $\left(\mu^{1}\right.$, $\mu^{2}$ below) are, independently of the specific utilities $U^{1} \neq U^{2}$, can be solved by the tool of derived orderings of tradeoffs.

DEFINITION 17. An outcome $\mu$ is called the midpoint-outcome between outcomes $\alpha, \beta$ if $[\alpha ; \mu] \sim{ }^{*}[\mu ; \beta]$; it is the midpoint-outcome between lotteries $P$ and $Q$ if it is the midpoint-outcome between certainty-equivalents $\mathrm{CE}(P) \sim P$ and $\mathrm{CE}(Q) \sim Q$. That is,

$$
[C E(P) ; \mu] \sim{ }^{*}[\mu ; C E(Q)] \text {. }
$$

The following lemma, a direct consequence of (5), shows that a midpoint-outcome is uniquely determined given the strict increasingness of utility.

LEMMA 18. Under $R D U$, if $\mu$ is the midpoint-outcome between $\alpha$ and $\beta$, then $U(\mu)=(U(\alpha)+U(\beta)) / 2$; if $\mu$ is the midpoint-outcome between $P$ and $Q$, then $U(\mu)=(R D U(P)+R D U(Q)) / 2$.

DEFINITION 19. We say that $\geq^{2}$ is more averse to probabilistic risk than $\geq^{1}$ if, for all $p, q, \lambda \in[0,1]$,

$$
\mu^{1} \geq^{1} \lambda P+(1-\lambda) Q \Rightarrow \mu^{2} \geq^{2} \lambda P+(1-\lambda) Q
$$

where $P=(p, M ; 1-p, 0), Q=(q, M ; 1-q, 0)$, and $\mu^{1}$ and $\mu^{2}$ are midpoint outcomes between $P$ and $Q$ of the respective decision makers. We say that $\geq^{2}$ is more prone to probabilistic risk if in (11) we have $\leq$ instead of $\geq ; \geq^{2}$ is equally averse to probabilistic risk if it is both more averse and more prone to probabilistic risk.

The antecedent preference in Equation (11) shows that the first decision maker prefers the midpoint-outcome $\mu^{1}$ to the ('two-stage') lottery that results with probability $\lambda$ in the lottery giving with 
probability $p$ the best outcome, and with probability $1-\lambda$ in the lottery giving with probability $q$ the best outcome. Then the second decision maker who has greater aversion to probabilistic mixing, should certainly prefer his midpoint-outcome to the increased probabilistic risk of the two-stage lottery.

In the above condition one could take any outcomes $\alpha>\beta$ instead of $M, 0$, and let these outcomes be different for the different decision makers. Thus the condition can even be used to characterize greater aversion to probabilistic risk if the outcome sets for the decision makers are different.

We rewrite the above condition directly in terms of preferences, to show its empirical content. First note that, under RDU, for a general preference relation $\geq$,

$$
\begin{aligned}
& {[\varphi(\lambda p+(1-\lambda) q) \text { is less/equal the midpoint between } \varphi(p) \text {, }} \\
& \quad \varphi(q)]
\end{aligned}
$$

is revealed by the preferences in Table 1 .

That is, certainty equivalents $\operatorname{CE}(P), \operatorname{CE}(Q)$ must be found for $P=(p, M ; 1-p, 0)$ and $Q=(q, M ; 1-q, 0)$ to give (i) and (ii). Next the midpoint outcome $\mu$ is found, by eliciting $[\mathrm{CE}(P) ; \mu] \sim{ }^{*}[\mu ; \mathrm{CE}(Q)]$; the equivalences in (iii) and (iv) are only simple examples of prefences to elicit this $\sim^{*}$ relation. It can be seen that $r, x, y$ as in (iii) and (iv) can always be found, mainly by choosing $r$ sufficiently large; many other sorts of preferences could be used instead to elicit the midpoint outcome. These were the 'structural', preparatory, preferences in Definition 19. Then, finally, (v) is the revealing preference.

If $\geq^{2}$ is more averse to probabilistic risk than $\geq^{1}$, then, whenever for $p, q, \lambda$ the preferences of Table 1 can be constructed to give (v) for

\section{TABLE 1}

Preferences revealing $\varphi(\lambda p+(1-\lambda) q) \leqslant \frac{\varphi(p)+\varphi(q)}{2}$.

$$
\begin{aligned}
& \mathrm{CE}(P) \sim P=(p, M ; 1-p, 0) . \\
& \mathrm{CE}(Q) \sim Q=(q, M ; 1-q, 0), \\
& (r, x ; 1-r, \mathrm{CE}(P)) \sim(r, y ; 1-r, \mu), \\
& (r, x ; 1-r ; \mu) \sim(r, y ; 1-r, \mathrm{CE}(Q)), \quad \text { and } \\
& \mu \geq \lambda P+(1-\lambda) Q .
\end{aligned}
$$


$\geq$, i.e., the antecedent preference in (11), it should also be possible to construct the similar preferences for $\geq^{2}$ (where the CEs and $\mu$ will of course be different).

THEOREM 20. Suppose that RDU holds for $\geq^{1}, \varphi^{1}, U^{1}$ as well as $\geq^{2}, \varphi^{2}, U^{2}$, with $\varphi^{1}$ and $\varphi^{2}$ continuous. Then $\varphi^{2}=\psi \circ \varphi^{1}$ for $a$ continuous, convex, strictly increasing $\psi:[0,1] \rightarrow[0,1]$ if and only if $\geq^{2}$ is more averse to probabilistic risk than $\geq{ }^{1}$. 'Convex' can be replaced by 'concave' if 'averse' is replaced by 'prone'; $\psi$ is the identity if and only if $\geq^{2}$ is equally risk averse.

Note that, by means of equal probabilistic risk aversion, identical attitudes towards probabilistic risk can be characterized in general, even if the utilities are not identical. As a corollary of the above result, convexity and concavity of $\varphi$ can again be characterized, independently of utility. This is done by comparison to the preferences of a linear $\varphi$, i.e., of 'classical' EU with linearity in probabilities. Linearity of $\varphi$ is characterized by equivalence in the antecedent preference in (11) for $\lambda=1 / 2$. Thus a condition to characterize convexity of $\varphi$ is:

For all probabilities $p, q$

$$
\mu \geq(P+Q) / 2
$$

for the midpoint-outcome $\mu$ of $P=(p, M ; 1-p, 0)$ and $Q=(q, M ; 1-$ $p, 0)$.

A decision maker, averse to probabilistic risk, will typically 'undervalue' the mixed lottery $(P+Q) / 2$ and assign to it a value less than the midpoint of the RDU values of the other two lotteries.

COROLLARY 21. Under RDU, $\varphi$ is convex if and only if (12) holds, it is concave if and only if (12) holds with $\leq$ instead of $\geq$, and $\varphi$ is linear if and only if (12) holds with $\sim$ instead of $\geq$.

Note that the last part of the above Corollary, together with Theorem 12, gives a characterization of EU maximization, alternative to, but of course far less attractive than, the one given by von Neumann and Morgenstern (1944), for continuous increasing utility. 
We could have introduced the term 'aversion to probabilistic risk' for (12), with terms risk prone and risk neutrality similar. The following subsection will, however, give more appealing conditions, therefore no name is reserved for the present conditions.

Convexity of $\varphi$ implies that (12) can be extended to general probability distributions $\mathrm{P}, \mathrm{Q}$. This can be derived from Observation 2 in a straightforward way, and is left to the reader:

OBSERVATION 22. In Corollary 21, Condition (12) can be replaced by the stronger condition:

For all probability distributions $P, Q$ and midpoint-outcomes $\mu$ between $P$ and $Q$, we have $\mu \geq(P+Q) / 2$.

The variations of (12) with $\leq$ and $\sim$ can be adapted similarly.

A similar strengthening is not possible for the more averse/prone-toprobabilistic-risk condition. In the definition of comparative probabilistic risk it is crucial that only probability distributions $P, Q$ are considered with one highest outcome, one lowest outcome, and no other outcomes. If $P, Q$ have three or more outcomes, then there may be different proportions of utility differences for the different decision makers and these can be seen to 'disturb' the relevation of probabilistic risk attitudes.

\subsection{A Natural Characterization of Probabilistic Risk Aversion}

This subsection characterizes probabilistic risk aversion directly through quasi-convexity of preference with respect to probabilistic mixing. This is an appealing and natural result, directly reflecting aversion to probabilistic mixing. Chew (1989, Corollary 2) already showed that convexity of the probability transformation $\varphi$ implies quasi-convexity of preference; the derivation of this result is elementary, see Observation 2. It is surprising that the reversed implication has not been obtained before in the literature. Indeed, its proof is not elementary. It shows that quasi-convexity of RDU implies convexity with respect to probability mixtures. 
The proof uses techniques developed in the study of quasi-concave additively decomposable functions (which are 'almost' concave). See for instance Yaari (1977), Debreu and Koopmans (1982), Crouzeix and Lindberg (1986), and Wakker (1989a, Sections VII.3 and VII.4). In the present context of RDU, quasi-convexity with respect to probability mixtures is interpreted as aversion to probabilistic risk, quasi-concavity as proneness, and their combination, betweenness (linearity) as neutrality.

DEFINITION 23. The binary relation $\geq$ is quasi-convex (with respect to probability mixtures) if $P \geq Q$ implies $P \geq \lambda P+(1-\lambda) Q$ for all $0<\lambda<1$, it is quasi-concave (with respect to probability mixtures) if $P \leq Q$ implies $P \leq \lambda P+(1-\lambda) Q$ for all $0<\lambda<1$, and it satisfies betweenness (or is linear with respect to probability mixtures) if it is both quasi-convex and quasi-concave.

THEOREM 24. Under RDU, $\varphi$ is convex if and only if $\geq$ is quasiconvex, $\varphi$ is concave if and only if $\geq$ is quasi-concave, $\varphi$ is linear if and only if $\geq$ satisfies betweenness.

It may seem paradoxical that probabilistic risk aversion, a reflection of pessimism, has been characterized through aversion to probabilistic mixtures, whereas in Schmeidler (1989, Remark 3), Gilboa and Schmeidler (1989), and Wakker (1990b) the pessimistic uncertainty aversion was characterized by appreciation of probabilistic mixtures. The explanation is as follows. In these references risk neutrality with respect to purely probabilistic risk is presupposed, contrary to the probabilistic risk aversion as characterized in the above theorem. Pessimism concerns nonprobabilized uncertainty there, as opposed to probabilized. As soon as there is nonprobabilized uncertainty, additional probabilistic mixing increases the role of probabilized risk with respect to which the decision maker is neutral, and decreases the role of nonprobabilized uncertainty with respect to which the decision maker is pessimistic. Thus he will appreciate probabilistic mixing. 


\section{THE MAIN THEOREM}

The following theorem combines all results of this paper, except the interpersonal comparability results in Theorems 14 and 20. Appendix 2 describes the extension to multidimensional, even nonquantitative, outcomes. The only remaining restrictive condition then is continuity of utility.

Next references are given to the definitions in this paper. The certainty-equivalent condition has been defined in Subsection 3.3, simple continuity in Definition 10, stochastic dominance in Assumption 3 , tradeoff consistency in Definition 6; decreasing, increasing, and constant marginal utility are given in Definition 15 , continuity in probability in Definition 11 , and quasi-convexity, quasi-concavity, and betweenness of $\geq$ in Definition 23 .

THEOREM 25 (Main theorem). Let $\geq$ be a preference relation on the set $\mathscr{P}$ of probability distributions over $[0, M]$. The following two statements are equivalent:

(i) RDU holds.

(ii) The preference relation $\geq$ is a weak order, it satisfies stochastic dominance, the certainty-equivalent condition, simple-continuity, and tradeoff consistency.

The probability transformation $\varphi$ in Statement $(i)$ is uniquely determined, the utility $U$ is cardinal. The following equivalences hold for $R D U$ :

(a) $U$ is concave $\Leftrightarrow \geq$ exhibits decreasing marginal utility;

(b) $U$ is convex $\Leftrightarrow \geq$ exhibits increasing marginal utility;

(c) $U$ is linear $\Leftrightarrow \geq$ exhibits constant marginal utility;

(d) $\varphi$ is continuous on the interval ]0,1[ (or at 0 , or at 1, respectively) $\Leftrightarrow \geq$ is continuous in probability on the interval $] 0,1[$ (or at 0 , or at 1 );

(e) $\varphi$ is convex $\Leftrightarrow \geq$ is quasi-convex;

(f) $\varphi$ is concave $\Leftrightarrow \geq$ is quasi-concave;

(g) $\varphi$ is linear $\Leftrightarrow \geq$ satisfies betweenness. 


\section{APPENDIX 1: PROOFS}

Often, without further mention, the result is used that a nondecreasing function from an interval to $\mathbb{R}$ is convex as soon as it is nondecreasing and satisfies 'midpoint-convexity', i.e., $\varphi((\mu+\nu) / 2) \leqslant(\varphi(\mu)+\varphi(\nu)) /$ 2 for all arguments $\mu, \nu$. This follows from Hardy, Littlewood and Pólya (1934, Theorems 111 and 86, where one should note that their term 'convex' has the same meaning as our term 'midpoint-convex'), firstly on the interior, then by nondecreasingness at a right endpoint, next by midpoint-convexity at a left endpoint.

Proof of Theorem 12. We first suppose that RDU holds and derive the conditions for the preference relation. With the exception of stochastic dominance, everything is straightforward from substitution and Equations (4) and (6). So we only derive stochastic dominance; it is taken in the strict sense in this paper. As is well known, stochastic dominance is satisfied if $\varphi$ is continuous. If $\varphi$ is not continuous, then the transformed decumulative distribution functions may be discontinuous and correspond to probability distributions that are not countably additive/set-continuous. Contrary to what has sometimes been thought, stochastic dominance need not hold under finite additivity, such as resulting for instance from Savage's (1954) model; this is pointed out in Wakker (1993d). However, in the present case, where $\mathscr{P}$ contains only countably additive probability distributions with rightcontinuous distribution functions, the fact that the transformations of these distribution functions may not be right-continuous does not pose a problem: suppose that $P$ stochastically dominates $Q$ and $P \neq Q$. Then there exists $\mu \in \mathbb{R}$ such that $P(U \leqslant \mu)<Q(U \leqslant \mu)$. Obviously, $\mu<U(M)$. By countable additivity and the implied right continuity of distribution functions, there exist $\delta, \epsilon>0$ such that $Q(U \leqslant \mu)-\epsilon>$ $P(U \leqslant \mu+\delta)$. For each $\mu<\tau<\mu+\delta$ we have $Q(U<\tau) \geqslant Q(U \leqslant$ $\mu)>Q(U \leqslant \mu)-\epsilon>P(U \leqslant \mu+\delta) \geqslant P(U<\tau)$. Hence $Q(U \geqslant \tau) \leqslant$ $Q(U>\mu)<Q(U>\mu)+\epsilon<P(U>\mu+\delta) \leqslant P(U \geqslant \tau)$. Applying (1) to calculate $\operatorname{RDU}(P)$ and $\operatorname{RDU}(Q)$, we see that the integrand for $P$ is always greater/equal than the integrand for $Q$ by stochastic dominance and countable additivity. On the interval $] \mu, \mu+\delta[$ the integrand for $P$ is greater/equal $\varphi(Q(U>\mu)+\epsilon)$, the integrand for $Q$ is less/equal $\varphi(Q(U>\mu))$. Because $\varphi$ is strictly increasing, strict prefer- 
ence of $P$ over $Q$ follows, as required by stochastic dominance. In more general contexts, where $\mathscr{P}$ may contain strictly finitely additive probability measures, stochastic dominance does not hold in general. As pointed out in Wakker (1993d), it should then be required only for simple probability distributions, with weak stochastic dominance imposed on all probability distributions.

Next suppose that the conditions for preferences hold. It must be shown that RDU holds. The proof uses the generalized results on additive representation theory as developed in Wakker (1991a, 1993a). First fix a probability vector $\left(p_{1}, \ldots, p_{n}\right)$ with $n \geqslant 2$. We simply write $\geq$ for the induced binary relation on $[0, M]_{\downarrow}^{n}$ (this notation was defined in Equation 7). This preference relation satisfies all conditions in Statement (ii) of Theorem 3.2 in Wakker (1993a): It is a weak order, continuous by simple-continuity, and it satisfies $\alpha \geqslant \beta \Leftrightarrow\left(x_{1}, \ldots, x_{i-1}\right.$, $\left.\alpha, x_{i+1}, \ldots, x_{n}\right) \geq\left(x_{1}, \ldots, x_{i-1}, \beta, x_{i+1}, \ldots, x_{n}\right) \quad$ ('monotonicity'). Tradeoff consistency immediately implies 'generalized triple cancellation', i.e., the condition of Observation 8 with $i=j$ and probabilities fixed. Continuity of $\geq$ on $[0, M]$ is immediate, $\geq$ being identical to $\geqslant$. By Theorem 3.2 of Wakker (1993a) there must exist an extended additive representation $\left(x_{1}, \ldots, x_{n}\right) \mapsto \sum_{j=1}^{n} V_{j}\left(x_{j}\right)$ for $\geq$ where possibly $V_{1}$ assigns the value $-\infty$ to the minimal outcome 0 and/or $V_{n}$ assigns the value $\infty$ to the maximal outcome $M=\infty$. All other $V_{j}$ values are real. By standard procedures it can be shown that tradeoff consistency implies that the different $V_{j}$ s 'locally' order differences the same way where they are finite and hence can be taken proportional to each other. This is completely similar to Wakker (1989a, Lemma VI.8.2) and will not be elaborated. It follows that, for the minimal outcome 0 , $\lim _{\tau \downarrow 0} V_{1}(\tau)$ is finite by proportionality with $V_{2}$ and that $V_{1}(0)$ can be taken as this limit; similarly $V_{n}(\mathrm{M})$ can be taken as real limit, and all $V_{j}$ s are real-valued and proportional on their entire domain. Consequently $\geq$ on $[0, M]_{\downarrow}^{n}$ is represented by $\left(x_{1}, \ldots, x_{n}\right) \mapsto \Sigma_{j=1}^{n} \lambda_{j} U\left(x_{j}\right)$ for a continuous cardinal $U$ and positive $\lambda_{j}$ s summing to one. Define $\varphi\left(\Sigma_{j=1}^{i} p_{j}\right):=\Sigma_{j=1}^{i} \lambda_{j}$ for all $1 \leqslant i \leqslant n$. By the uniqueness up to similar positive affine transformations of the $V_{j}$ s found above (see Wakker, 1993a, 'joint cardinality'), those $\varphi$ values are uniquely determined and $U$ is cardinal. 
At this stage the $U$ and $\varphi$ values obtained might be thought to depend on the chosen probability vector $\left(p_{1}, \ldots, p_{n}\right)$. However, for any two probability vectors a third 'more refined' probability vector can be considered such that all lotteries obtained from the first or second probability vector can also be obtained from the third. By standard arguments this shows that the $\varphi$ values are independent of the particular partition, and that $\mathrm{U}$ is cardinal and can also be chosen independent of the partition. In short, the desired RDU representation and uniqueness results have been obtained for the simple lotteries.

The extension to nonsimple lotteries is routine and, given the minimum and maximum utility values, an elementary application of Wakker (1993c). A brief sketch is as follows. Because each considered probability distribution $P$ is bounded (by $0, M$ ), there exist sequences of simple probability distributions $\left(P^{j}\right)_{j=1}^{\infty}$ and $\left(Q^{j}\right)_{j=1}^{\infty}$ such that the $P^{j}$ s are all stochastically dominated by $P$ and the $Q^{j}$ s all stochastically dominate $P$, and further $1 / j \geqslant G_{Q^{j}, U}^{-1}(\tau)-G_{P^{j}, U}^{-1}(\tau) \geqslant 0$ for all $j, \tau$, with the inverses of distribution functions defined on $[0,1]$ as usual. This uniquely determines the $U$ value of the certainty equivalent of $P$, which represents preferences, as the RDU value of $P$.

That continuity conditions of $\varphi$ imply the corresponding continuity conditions in probability for $\geq$ is straightforward. Next suppose that (8) holds. Take an arbitrary $0<p<1$ and let $(p, M ; 1-p, 0) \sim \beta$. Taking $\beta_{j}$ convergent to $\beta$ from below (for more general outcome sets, as considered in Appendix 2, let $U\left(\beta_{j}\right)$ converge to $U(\beta)$ from below) and applying (8), shows that $\varphi$ is left-continuous at $p$. The other continuity results are established similarly.

Proof of Theorem 14. The implication (i) $\Rightarrow$ (ii) follows from the lines below the theorem. So we assume (ii) and derive (i).

We take any fixed $0<p<1$ and use only lotteries of the form $\left(p, x_{1}\right.$; $\left.1-p, x_{2}\right)$. Suppose that $\mu$ is a nonextreme outcome. The next paragraph shows that an interval $S:=\left\{\alpha \in \mathscr{C}: \mu^{+} \geqslant \alpha \geqslant \mu^{-}\right\}$, with $\mu^{+}>\mu>\mu^{-}$, can be found so that on $S$ the implication in (4) can be reversed for $\geq^{1}$.

The details for finding $S$ are as follows. Take any $\theta<\mu$, e.g. $\theta=0$. By connectedness of the range of $U$ we can take $\mu^{+}, \mu^{-}$so 'close' to $\mu$ that $\mu^{+}>\mu>\mu^{-}>\theta$ and $\varphi^{1}(p)\left(U^{1}\left(\mu^{+}\right)-U^{1}\left(\mu^{-}\right)\right)<\left[1-\varphi^{1}(p)\right]$ 
$\left(U^{1}\left(\mu^{-}\right)-U^{1}(\theta)\right)$. Then for each $\alpha>\beta$ in $S:=\left\{\gamma: \mu^{+} \geqslant \gamma \geqslant \mu^{-}\right\}$, $(p, \alpha ; 1-p, \theta)<^{1}\left(p, \beta ; 1-p, \mu^{-}\right)$. Obviously, $(p, \alpha ; 1-p, \theta)>^{1}$ $(p, \beta ; 1-p, \theta)$. By continuity and connectedness, there exists $\sigma(\alpha, \beta)$ between $\mu^{-}$and $\theta$ such that $(p, \alpha ; 1-p, \theta) \sim{ }^{1}(p, \beta ; 1-p, \sigma(\alpha, \beta))$. Hence for each $\alpha>\beta, \gamma>\delta$ in $S, U^{1}(\alpha)-U^{1}(\beta) \geqslant U^{1}(\gamma)-$ $U^{1}(\delta) \Rightarrow(p, \gamma ; 1-p, \theta) \leq^{1}(p, \delta ; 1-p, \sigma(\alpha, \beta)) \Rightarrow \alpha \beta \geq^{1 *} \gamma \delta$ (the last implication uses the $\sim^{1}$ equivalence above).

Hence on $S$, for all $\alpha \geqslant \beta \geqslant \gamma \geqslant \delta, U^{1}(\alpha)-U^{1}(\beta) \leqslant U^{1}(\gamma)-U^{1}(\delta)$ implies $\alpha \beta \leq^{1 *} \gamma \delta$, which implies, by stronger decrease of marginal utility, $\alpha \beta \leq^{2 *} \gamma \delta$. This implies, by (4), $U^{2}(\alpha)-U^{2}(\beta) \leqslant U^{2}(\gamma)-$ $U^{2}(\delta)$. By standard arguments, Statement (i) is satisfied on the open interval $U^{1}(S)$ around the point $U^{1}(\mu)$ in the interior of the range of $U^{1}$. The result follows on $U^{1}(] 0, M[)$, and by continuity on the entire $U^{1}([0, M])$.

Proof of Theorem 20. We only derive the results concerning convexity. First suppose that $\varphi^{2}=\psi \circ \varphi^{1}$ with $\psi$ convex, and obviously continuous and strictly increasing. Suppose that $p, q, \lambda, \mu^{1}$ are as in the antecedent preference in (11). By connectedness of the range of $U^{2}$ one straightforwardly obtains the midpoint-outcome $\mu^{2}$ between $P$ and $Q$. For simplicity of notation, set $U^{1}(0)=U^{2}(0)=0, U^{1}(M)=$ $U^{2}(M)=1$. Substituting RDU and Lemma 18 in the antecedent preference in (11) gives

$$
\frac{\varphi^{1}(p)+\varphi^{1}(q)}{2} \geqslant \varphi^{1}(\lambda p+(1-\lambda) q) .
$$

So

$$
\begin{aligned}
\frac{\varphi^{2}(p)+\varphi^{2}(q)}{2} & =\frac{\psi\left(\varphi^{1}(p)\right)+\psi\left(\varphi^{1}(q)\right)}{2} \\
& \geqslant \psi\left(\frac{\varphi^{1}(p)+\varphi^{1}(q)}{2}\right) \\
& \geqslant \psi\left(\varphi^{1}(\lambda p+(1-\lambda) q)\right) \\
& =\varphi^{2}(\lambda p+(1-\lambda) q),
\end{aligned}
$$

the first inequality by convexity of $\psi$, the second by (13). The 
established inequality implies the second preference in (11). So $\geq^{2}$ is more averse to probabilistic risk than $\geq^{1}$.

Next we suppose that $\geq^{2}$ is more averse to probabilistic risk than $\geq^{1}$ and derive $\psi$ as above. As $\varphi^{1}$ and $\varphi^{2}$ are strictly increasing and continuous functions from $[0,1]$ to $[0,1]$, there exists a $\psi$ with all desired properties except possibly convexity; convexity is established next. For each $p, q$ there exists, by continuity of $\varphi$, a $\lambda$ such that $\varphi^{1}(\lambda p+(1-\lambda) q)=\left(\varphi^{1}(p)+\varphi^{1}(q)\right) / 2$. This gives the antecedent preference in (11) (with equivalence), which implies the second preference in (11), thus the right-hand side of (9). It follows that $\psi$ satisfies the midpoint-convexity condition for its arguments $\varphi^{1}(p)$, $\varphi^{1}(q)$, hence, by surjectivity of $\varphi^{1}$, for all of its arguments.

Proof of Corollary 21. We only consider the case of convexity. Setting $U(M)=1, U(0)=0$, the condition in $(12)$ implies $(\varphi(p)+$ $\varphi(q)) / 2 \geqslant \varphi(p+q) / 2$, i.e., midpoint-convexity. Convexity follows. The reversed implication, even for general lotteries $P, Q$, follows from Observation 2.

Proof of Theorem 24. We only consider the first statement. As shown in Observation 2, convexity of $\varphi$ implies convexity of RDU, hence quasi-convexity of $\geq$. So assume that $\geq$ is quasi-convex, which implies that RDU is also quasi-convex. It remains to be proved that $\varphi$ is convex, i.e., RDU is convex.

The 'leading idea' in the proof below is based on a measure for convexity, different from the Pratt/Arrow measure and 'vanishing in the small'. For any $\nu>\mu$ we write

$$
\lambda[\mu, \nu]:=\frac{\varphi(\mu) / 2+\varphi(\nu) / 2-\varphi(\mu / 2+\nu / 2)}{\varphi(\nu)-\varphi(\mu)} .
$$

This $\lambda$ can be interpreted as a measure of convexity of $\varphi$ on the interval $[\mu, \nu]$. Suppose that the strictly increasing $\varphi$ is nonconvex. Then some interval $[\mu, \nu]$ has a negative $\lambda$ value. Loosely speaking, for any interval $[\sigma, \tau]$ disjoint from $[\mu, \nu]$, it will be shown, by consideration of proper lotteries, that, under some restrictions, the measure of convexity of $\varphi$ with respect to $[\sigma, \tau]$ must kind of make up for $[\mu, \nu]$, 
and be greater/equal than $-\lambda$. Since the mentioned measure will (under differentiability) tend to zero as $\tau$ tends to $\sigma$, nonconvexity of $\varphi$ leads to a contradiction. A detailed elaboration is given below.

As a preparation for the proof of the claim about $-\lambda$ above, we show, for general $\mu, \nu, \sigma, \tau$ :

LEMMA 26. Suppose that $\mu<\nu \leqslant \sigma<\tau$. Then $\lambda[\mu, \nu]+\lambda[\sigma, \tau] \geqslant 0$.

Proof. All lotteries in this proof are of the form $\left(\mu, x_{1} ;(\nu-\mu) / 2\right.$, $\left.x_{2} ;(\nu-\mu) / 2, x_{3} ;(\sigma-\nu), x_{4} ;(\tau-\sigma) / 2, x_{5} ;(\tau-\sigma) / 2, x_{6} ; 1-\tau, x_{7}\right)$, with fixed probabilities and $x_{1} \geqslant \cdots \geqslant x_{7}$, and always with $x_{1}=\alpha$, $x_{4}=\gamma, x_{7}=\epsilon .^{10}$ So the seven probabilities are suppressed. As an aid for the reasonings below, we display the seven decision weights (defined in (3)):

$$
\begin{aligned}
& \varphi(\mu), \varphi\left(\frac{\nu+\mu}{2}\right)-\varphi(\mu), \varphi(\nu)-\varphi\left(\frac{\nu+\mu}{2}\right), \varphi(\sigma)-\varphi(\nu), \\
& \varphi\left(\frac{\sigma+\tau}{2}\right)-\varphi(\sigma), \varphi(\tau)-\varphi\left(\frac{\sigma+\tau}{2}\right), 1-\varphi(\tau)
\end{aligned}
$$

We can take outcomes $\alpha>\beta \geqslant \gamma \geqslant \delta>\epsilon$ such that

$$
(\alpha, \beta, \beta, \gamma, \delta, \delta, \epsilon) \sim(\alpha, \alpha, \alpha, \gamma, \epsilon, \epsilon, \epsilon)
$$

Calculations are greatly simplified by working with $\mathrm{RDU}^{*}$, instead of RDU, with

$$
\begin{aligned}
\operatorname{RDU}^{*}:= & \operatorname{RDU}-\operatorname{RDU}((\alpha, \beta, \beta, \gamma, \epsilon, \epsilon, \epsilon)) \\
= & \operatorname{RDU}-\left\{\varphi(\mu) U(\alpha)+\left[\varphi\left(\frac{\nu+\mu}{2}\right)-\varphi(\mu)\right] U(\beta)\right. \\
& +\left[\varphi(\nu)-\varphi\left(\frac{\nu+\mu}{2}\right)\right] U(\beta)+[\varphi(\sigma)-\varphi(\nu)] U(\gamma) \\
& +\left[\varphi\left(\frac{\tau+\sigma}{2}\right)-\varphi(\sigma)\right] U(\epsilon) \\
& \left.+\left[\varphi(\tau)-\varphi\left(\frac{\tau+\sigma}{2}\right)\right] U(\epsilon)+[1-\varphi(\tau)] U(\epsilon)\right\}
\end{aligned}
$$

That is, we cancel common first, fourth, and seventh, terms, and 
subtract second, third, fifth, and sixth terms as minimal in (15). The equivalence (15) in terms of RDU* equalities gives

$$
[\varphi(\tau)-\varphi(\sigma)](U(\delta)-U(\epsilon))=[\varphi(\nu)-\varphi(\mu)](U(\alpha)-U(\beta))
$$

By quasi-convexity of $\geq$ each of the two lotteries in (15) is preferred to the $\frac{1}{2} / \frac{1}{2}$ mixture of them, i.e., $(\alpha, \alpha, \beta, \gamma, \delta, \epsilon, \epsilon)$. So each of their RDU $^{*}$ values, as well as the $\frac{1}{2} / \frac{1}{2}$ mixture of them, is at least as large as the $\mathrm{RDU}^{*}$ of the mixture-lottery:

$$
\begin{gathered}
{\left[\frac{\varphi(\nu)-\varphi(\mu)}{2}\right](U(\alpha)-U(\beta))} \\
+\left[\frac{\varphi(\tau)-\varphi(\sigma)}{2}\right](U(\delta)-U(\epsilon)) \geqslant \\
{\left[\varphi\left(\frac{\nu+\mu}{2}\right)-\varphi(\mu)\right](U(\alpha)-U(\beta))} \\
+\left[\varphi\left(\frac{\tau+\sigma}{2}\right)-\varphi(\sigma)\right](U(\delta)-U(\epsilon)) .
\end{gathered}
$$

Subtracting gives

$$
\begin{aligned}
& {\left[\frac{\varphi(\nu)+\varphi(\mu)}{2}-\varphi\left(\frac{\nu+\mu}{2}\right)\right](U(\alpha)-U(\beta))} \\
& \quad+\left[\frac{\varphi(\tau)+\varphi(\sigma)}{2}-\varphi\left(\frac{\tau+\sigma}{2}\right)\right](U(\delta)-U(\epsilon)) \geqslant 0 .
\end{aligned}
$$

Dividing by the positive factors in (16) gives

$$
\begin{aligned}
& {\left[\frac{\varphi(\nu)+\varphi(\mu)}{2}-\varphi\left(\frac{\nu+\mu}{2}\right)\right] /[\varphi(\nu)-\varphi(\mu)]} \\
& \quad+\left[\frac{\varphi(\tau)+\varphi(\sigma)}{2}-\varphi\left(\frac{\tau+\sigma}{2}\right)\right] /[\varphi(\tau)-\varphi(\sigma)] \geqslant 0 .
\end{aligned}
$$

So $\lambda[\mu, \nu]+\lambda[\sigma, \tau] \geqslant 0$.

Suppose now that the strictly increasing $\varphi$ is not convex. Then for some interval, say $[\mu, \nu]$, we have $\lambda[\mu, \nu]<0$. It follows from the above lemma that $\varphi$ is convex on $[0, \mu]$ and $[\nu, 1]$. In particular it has a 
finite right derivative everywhere on $[0, \mu[$ and $[\nu, 1[$. For each $\sigma$ from one of these intervals where $\varphi$ has positive right derivative, it follows from elementary analysis that $\lambda[\sigma, \tau]$ tends to 0 if $\tau$ decreases to $\sigma$, the derivative being finite. This contradicts Lemma 26 for a $\tau$ close enough to $\sigma$. Hence, everywhere on $[0, \mu[$ and on $[\nu, 1[$ the right derivative of $\varphi$ is 0 , and $\varphi$ must be constant. Because $\varphi$ is strictly increasing, $\mu=0$, $\nu=1$, and $\varphi(1 / 2)>1 / 2$ follows. Now take any $0<\sigma<1 / 2$. Then $\varphi$ is not convex on $[0,1 / 2]$ or on $[\sigma, 1]$, thus has an interval with negative $\lambda$ within one of these two intervals. But, as shown above, this either implies that $\varphi$ is constant on $[1 / 2,1[$, or on $[0, \sigma[$. Neither of these is possible. Nonconvexity of $\varphi$ is impossible.

\section{APPENDIX 2: EXTENSION TO GENERAL OUTCOME SETS}

For the sake of easy accessibility, in the main body of the paper the results have been formulated for the case where $\mathscr{C}$ is a bounded closed interval $[0, M]$, and utility is strictly increasing. This can straightforwardly be generalized to the case where $\mathscr{C}$ is any connected topological space, mainly by Wakker (1993c and 1993a). The proof for the case $\mathscr{C}=[0, M]$ has been presented with this in mind. The adaptation of the results is described briefly.

In general, strict increasingness of $U$ is deleted everywhere (in particular in the definition of RDU) and the usual ordering $\geqslant$ on $\mathscr{C}$ is replaced everywhere by the preference relation $\geq$ restricted to $\mathscr{C}$ (outcomes identified with degenerate lotteries), where there are assumed to exist outcomes $\alpha>\beta$. Equalities $\alpha=\beta$ are sometimes replaced by equivalences $\alpha \sim \beta$; the midpoint-outcomes in Definition 17 are no longer uniquely determined. Under RDU, $U$ obviously orders outcomes in agreement with $\geq$ on the outcomes, which gives the analogue of strict increasingness. The latter also gives natural extensions of stochastic dominance, similarly to Wakker (1990a; there events of obtaining a fixed outcome or any outcome at least as good were considered): $P$ stochastically dominates $Q$ if for each outcome $\alpha \in \mathscr{C}, P(\{\beta \in \mathscr{C}: \beta \leq \alpha\}) \leqslant Q(\{\beta \in \mathscr{C}: \beta \leq \alpha\})$. Several conditions in the text used lotteries $(p, M ; 1-p, 0)$; here one takes instead the lottery $(p, \sigma ; 1-p, \tau)$ for any outcomes $\sigma>\tau$.

With the above modifications, all results and proofs concerning 
probabilistic risk aversion in Subsections 4.2 and 4.3 remain valid without further modifications. In Definition 13, the comparative definition of decreasing marginal utility, the condition must be added that the two preferences coincide for the certain outcomes, and one should take $\alpha \geq^{1} \beta \geq^{1} \gamma \geq^{1} \delta$. With these modifications, Theorem 14 and its proof remain valid. Because there is no direct meaning for concavity/convexity/linearity of utility for general outcomes, no adaptation of Corollary 16 should have been expected.

So we finally turn to Theorem 12 . The result is adapted here only on the set of 'bounded lotteries'. A lottery is bounded if it assigns probability 1 to a set $\{\beta \in \mathscr{C}: \alpha \geq \beta \geq \gamma\}$. That all conditions for preferences follow from RDU is straightforward and is not elaborated. So suppose that all conditions for preferences hold. To derive is that RDU holds. Continuity of $\geq$ on $\mathscr{C}$ is now derived from simplecontinuity with $n=1$. Then the derivation of RDU for simple distributions is exactly as in the proof of Theorem 12. The extension of RDU to the bounded lotteries is obtained by the same reasoning as in the proof of Theorem 12, but now for the probability distributions as induced on the range of $U$. Also uniqueness results require no adaptation. The utility function may very well be unbounded, for example if $\mathscr{C}=\mathbb{R}$ and the utility function is the identity.

The extension to any arbitrary subset of the set of lotteries with finite RDU value, also if not bounded, can be obtained by Theorems 21 and 29 in Wakker (1993c), because an underlying probability space can always be constructed and RDU then is a special case of Choquet expected utility. For brevity, elaboration is omitted.

THEOREM 27. Let the outcome set $\mathscr{C}$ be an arbitrary connected topological space instead of $[0, M]$ and let $\mathscr{P}$ be the set of all bounded lotteries over $\mathscr{C}$. Then Theorem 12, Theorem 14 with the condition $\left[\alpha \geq^{1} \beta \Leftrightarrow \alpha \geq^{2} \beta\right]$ added in Statement (ii), Theorem 20, Corollary 21, Observation 22, Theorem 24, and Theorem 25 without Statements (a), (b), and (c), remain valid.

Finally, we give some additional comments on the simple continuity condition used in this paper. The most customary continuity conditions for probability distributions are continuity with respect to weak 
convergence, or with respect to $L_{1}$ convergence, of the distribution functions. This requires both continuity in outcomes, implying continuity of utility in RDU, and continuity in probabilities, which implies continuity of the probability transformation $\varphi$. It also imposes continuity restrictions on probability distributions with infinitely many outcomes; 'infinite-dimensional' continuity is complicated and more restrictive, and often implies boundedness of utility. As shown in this Appendix, Wakker (1989a, Chapter V), and Wakker (1993c), simple continuity does allow for unbounded utility.

\section{ACKNOWLEDGEMENTS}

The research has been made possible by a fellowship of the Royal Netherlands Academy of Arts and Sciences, and a fellowship of the Netherlands Organization for Scientific Research. The first version of this paper received helpful comments from Bob Nau and Rakesh Sarin, the present version from anonymous referees and numerous colleagues; Hein Fennema gave very useful comments.

\section{NOTES}

\footnotetext{
${ }^{1}$ A discussion of the results in rank-dependent utility that are presently available is given in the beginning of Section 4 .

${ }^{2}$ See the sixteenth paragraph (Let ... etc.) in combination with the ninteenth paragraph (The ... expectation) of Section 3.

${ }^{3}$ Friedman and Savage (1948) sometimes seemed to have a different point of departure; their work may be taken as evidence that risk attitudes cannot be entirely captured in utility.

${ }^{4}$ Compare Savage (1954, p. 101).

${ }^{5}$ Compare for instance Krzysztofowicz (1983, Conclusion 1) with Krzysztofowicz and Koch (1989, top of p. 182); see also the early McCord and de Neufville (1983, p. 295).

${ }^{6}$ The latter can be modeled by 'Choquet expected utility', as initiated by Schmeidler (1989) for decisions under certainty. Wakker (1990a) shows that RDU is the special case of Choquet expected utility when applied to decisions under risk, the domain of this paper. For a mixed case of risk and uncertainty, Jaffray (1989) developed a model that distinguishes between marginal utility and attitudes towards ambiguity, but preserves neutrality with respect to probabilistic risk.

${ }^{7}$ In RDU formalized through decision weights in Formula (3).

${ }^{8}$ By the conventions of notation of this paper, for $p=1$ we write $(1, M)$ rather than $(1, M ; 0,0)$, and for $p=0$ we write $(1,0)$ rather than $(0, M ; 1,0)$.

${ }^{9}$ Concavity in the dual setup of Chew, Karni, and Safra (1987).
} 
${ }^{10}$ Given the convention that all probabilities in the notations of lotteries should be positive, the seven-outcome notation above applies only if $0<\mu<\nu<\sigma<\tau<1$. If $0=\mu$ or $\nu=\sigma$ or $\tau=1$ then, strictly speaking, the associated outcomes $\alpha$ or $\gamma$ or $\epsilon$ should be suppressed. This does not affect the proof, where those fixed outcomes play no role anyhow. Therefore the conclusions also hold for those probabilities.

\section{REFERENCES}

Allais, M.: 1953, 'Fondements d'une Théorie Positive des Choix Comportant un Risque et Critique des Postulats et Axiomes de l'Ecole Américaine', Colloques Internationaux du Centre National de la Recherche Scientifique 40, Econométrie, 257-332. Paris: Centre National de la Recherche Scientifique. Translated into English, with additions, as 'The Foundations of a Positive Theory of Choice Involving Risk and a Criticism of the Postulates and Axioms of the American School, in Allais, M. and Hagen, O. (Eds.): 1979, Expected Utility Hypotheses and the Allais Paradox, Reidel, Dordrecht, The Netherlands, pp. 27-145.

Allais, M.: 1979, 'The So-Called Allais Paradox and Rational Decisions under Uncertainty', In Allais, M. and Hagen, O. (Eds.): 1979, Expected Utility Hypotheses and the Allais Paradox, Reidel, Dordrecht, The Netherlands, pp. 437-681.

Arrow, K.J.: 1951, 'Alternative Approaches to the Theory of Choice in Risk-Taking Situations', Econometrica 19, 404-437.

Barron, F.H., von Winterfeldt, D. and Fischer, G.W.: 1984, 'Empirical and Theoretical Relationships between Value and Utility Functions', Acta Psychologica 56, 233-244.

Bernard, G.: 1974, 'On Utility Functions', Theory and Decision 5, 205-242.

Bernoulli, D.: 1738, 'Specimen Theoria Novae de Mensura Sortis', Commentarii Academiae Scientiarum Imperialis Petropolitanae 5, 175-192. Translated into English by Sommer, L.: 1954, 'Exposition of a New Theory on the Measurement of Risk', Econometrica 12, 23-36; or in Page, A.N. (Ed.): 1968, Utility Theory: A Book of Readings, Wiley, New York, Ch. 11.

Birnbaum, M.H. and Sutton, S.E.: 1992, 'Scale Convergence and Utility Measurement', Organizational Behavior and Human Decision Processes 52, 183-215.

Camerer, C.F.: 1989, 'An Experimental Test of Several Generalized Utility Theories', Journal of Risk and Uncertainty 2, 61-104.

Chateauneuf, A.: 1988, 'Uncertainty Aversion and Risk Aversion in Models with Nonadditive Probabilities', In Munier, B.R. (Ed.): 1988, Risk, Decision and Rationality, Reidel, Dordrecht, pp. 615-629.

Chateauneuf, A.: 1990, 'On the Use of Comonotonicity in the Axiomatization of EURDP Theory for Arbitrary Consequences', CERMSEM, University of Paris I; Abstract presented at Fifth International Conference on the Foundations and Applications of Utility, Risk and Decision Theory (FUR-90).

Chateauneuf, A and Cohen, M.: 1990, 'Risk Secking with Diminishing Marginal Utility in a Non-Expected Utility Model', CERMSEM, University of Paris I.

Chew, S.H.: 1989, 'An Axiomatic Generalization of the Quasilinear Mean and Gini Mean with Application to Decision Theory', Department of Economics, University of California, Irvine, USA.

Chew, S.H. and Epstein, L.G.: 1989, 'A Unifying Approach to Axiomatic NonExpected Utility Theories', Journal of Economic Theory 49, 207-240. 
Chew, S.H., Epstein, L.G. and Wakker, P.P.: 1993, 'A Unifying Approach to Axiomatic Non-Expected Utility Theories: Corrigenda', Journal of Economic Theory 59, 183188.

Chew, S.H. and Karni, E.: 1991, 'Choquet Expected Utility with a Finite State Space', Journal of Economic Theory, forthcoming.

Chew, S.H., Karni, E. and Safra, Z.: 1987, 'Risk Aversion in the Theory of Expected Utility with Rank Dependent Probabilities', Journal of Economic Theory 42, 370-381.

Chew, S.H. and Wakker, P.P.: 1991, 'Generalizing Choquet Expected Utility by Weakening Savage's Sure-Thing Principle', University of California, Irvine Research Unit in Mathematical Behavioral Sciences, MBS 91-16, Irvine, CA, USA.

Cohen, M. and Jaffray, J.Y.: 1988, 'Preponderence of the Certainty Effect over Probability Distortion in Decision Making under Risk', In Munier, B.R. (Ed.): 1988, Risk, Decision and Rationality, Reidel, Dordrecht, pp. 173-187.

Cramer, G.: 1728, Letter from Cramer to Nicholas Bernoulli. Translated into English by Louise Sommer in Bernoulli, Daniel (1954), 'Exposition of a New Theory on the Measurement of Risk', Econometrica 22, 23-36.

Crouzeix, J.-P. and Lindberg, P.O.: 1986, 'Additively Decomposed Quasiconvex Functions', Mathematical Programming 35, 42-57.

Debreu, G. and Koopmans, T.C.: 1982, 'Additively Decomposed Quasiconvex Functions', Mathematical Programming 24, 1-38.

Dyer, J.S. and Sarin, R.K.: 1982, 'Relative Risk Aversion', Management Science 28, $875-886$.

Edwards, W.: 1962, 'Subjective Probabilities Inferred from Decisions', Psychological Review 69, 109-135.

Farquhar, P. and Keller, R.L.: 1989, 'Preference Intensity Measurement', Annals of Operations Research 19, 205-217.

Fishburn, P.C.: 1978, 'On Handa's 'New Theory of Cardinal Utility' and the Maximization of Expected Return', Journal of Political Economy 86, 321-324.

Fishburn, P.C.: 1989, 'Retrospective on the Utility Theory of von Neumann and Morgenstern', Joumal of Risk and Uncertainty 2, 127-158.

Fisher, I.: 1927, 'A Statistical Method for Measuring "Marginal Utility" and Testing the Justice of a Progressive Income Tax', In Hollander, J.H. (Ed.): 1927 Economic Essays Contributed in Honor of John Bates Clark, Macmillan, New York, pp. 157-193.

Friedman, M. and Savage, L.J.: 1948, 'The Utility Analysis of Choices Involving Risk', Journal of Political Economy 56, 279-304.

Gilboa, 1. and Schmeidler, D.: 1989, 'Maxmin Expected Utility with a Non-Unique Prior', Journal of Mathematical Economics 18, 141-153.

Green, J. and Jullien, B.: 1988, 'Ordinal Independence in Non-Linear Utility Theory', Journal of Risk and Uncertainty 1, 355-387; ('Erratum', 2 (1989), 119.)

Gul, F.: 1992, 'Savage's Theorem with a Finite Number of States', Journal of Economic Theory 57, 99-110.

Handa, J.: 1977, 'Risk, Probabilities, and a New Theory of Cardinal Utility', Journal of Political Economy 85, 97-122.

Hardy, G.H., Littlewood, J.E. and Pólya, G.: 1934, Inequalities, Cambridge University Press, Cambridge (second edition 1952, reprinted 1978).

Harsanyi, J.C.: 1955, "Cardinal Welfare, Individualistic Ethics, and Interpersonal Comparisons of Utility', Journal of Political Economy 63, 309-321.

Hilton, R.W.: 1988, 'Risk Attitude under Two Alternative Theories of Choice under Risk', Joumal of Economic Behaviour and Organization 9, 119-136. 
Jaffray, J.Y.: 1989, 'Linear Utility Theory for Belief Functions', Operations Research Letters 8, 107-112.

Jevons, W.S.: 1911, The Theory of Political Economy, MacMillan, London.

Kahneman, D. and Tversky, A.: 1979, 'Prospect Theory: An Analysis of Decision under Risk', Econometrica 47, 263-291.

Krantz, D.H., Luce, R.D., Suppes, P., and Tversky, A.: 1971, Foundations of Measurement, Vol. I.: Additive and Polynomial Representations, Academic Press, New York.

Krelle, W.: 1968, Präferenz- und Entscheidungstheorie, Mohr, Tübingen.

Krzysztofowicz, R.: 1983, 'Strength of Preferences and Risk Attitude in Utility Measurement', Organizational Behaviour and Human Performance 31, 88-113.

Krzysztofowicz, R. and Koch, J.B.: 1989, 'Estimation of Cardinal Utility Based on a Nonlinear Theory', Annals of Operations Research 19, 181-204.

Lopes, L.L.: 1987, 'Between Hope and Fear: The Psychology of Risk', Advances in Experimental Psychology 20, 255-295.

Luce, R.D.: 1988, 'Rank-Dependent, Subjective Expected-Utility Representations', Journal of Risk and Uncertainty 1, 305-332.

Luce, R.D. and Fishburn, P.C.: 1991, 'Rank- and Sign-Dependent Linear Utility Models for Finite First-Order Gambles', Journal of Risk and Uncertainty 4, $29-59$.

Luce, R.D. and Raiffa, H.: 1957, Games and Decisions, Wiley, New York.

McCord, M.R. and de Neufville, R.: 1983, 'Fundamental Deficiency of Expected Utility Decision Analysis', In French, S., Hartley, R., Thomas, L.C., and White, D.J. (Eds.): 1983, Multi-Objective Decision Making, Academic Press, New York, pp. 279-305.

Marshall, A.: 1948, Principles of Economics, 8th Edition, MacMillan, New York.

Munier B.R. and Abdellaoui, M.: 1991, 'Expected Utility Violations: An Appropriate and Intercultural Experiment', In Chikàn, A. (Ed.): 1991, New Developments in Risk and Utility Analysis, Kluwer Academic Publishers, Dordrecht (forthcoming).

Nakamura, Y: : 1992, 'Multi-Symmetric Structures and Non-Expected Utility', Joumal of Mathematical Psychology 36, 375-395.

Pfanzagl, J.: 1968, Theory of Measurement, Physica-Verlag, Vienna.

Preston, M.G. and Baratta, P.: 1948, 'An Experimental Study of the Auction Value of an Uncertain Outcome', American Journal of Psychology 61, 183-193.

Quiggin, J.: 1982, 'A Theory of Anticipated Utility', Journal of Economic Behaviour and Organization 3, 323-343.

Quiggin, J. and Wakker, P.P.: 1992, 'The Axiomatic Basis of Anticipated Utility; A Clarification', CenTER Discussion paper No. 9203, University of Brabant, Tilburg, The Netherlands.

Ramsey, F.P.: 1931, 'Truth and Probability'. In Ramsey, F.P.: 1931, The Foundations of Mathematics and other Logical Essays, Routledge and Kegan Paul, London, pp. 156-198. Reprinted in Kyburg, H.E, and Smokler, H.E. (Eds.): 1964, Studies in Subjective Probability, Wiley, New York, pp. 61-92.

Savage, L.J.: 1954, The Foundations of Statistics, Wiley, New York (second edition 1972, Dover, New York).

Schmeidler, D.: 1989, 'Subjective Probability and Expected Utility without Additivity, Econometrica 57, 571-587.

Segal, U.: 1987, "The Ellsberg Paradox and Risk Aversion: An Anticipated Utility Approach', International Economic Review 28, 175-202. 
Segal, U.: 1989, 'Anticipated Utility: A Measure Representation Approach', Annals of Operations Research 19, 359-373.

Segal, U.: 1990, 'Two-Stage Lotteries without the Reduction Axiom', Econometrica 58, 349-377.

Segal, U.: 1993, 'The Measure Representation: A Correction', Journal of Risk and Uncertainty 6, 99-107.

Tversky, A. and Kahneman, D.: 1992, 'Advances in Prospect Theory: Cumulative Representation of Uncertainty', Journal of Risk and Uncertainty 5, 297-323.

von Neumann, J. and Morgenstern, O.: 1944, 1947, 1953, Theory of Games and Economic Behavior, Princeton University Press, Princeton, NJ.

von Winterfeldt, D. and Edwards, W.: 1986, Decision Analysis and Behavioral Research, Cambridge University Press, Cambridge.

Wakker, P.P.: 1984, 'Cardinal Coordinate Independence for Expected Utility', Journal of Mathematical Psychology 28, 110-117.

Wakker, P.P.: 1986, 'Representations of Choice Situations', Ph.D. Disseration, University of Brabant, Department of Economics, The Netherlands.

Wakker, P.P.: 1987, 'Nonadditive Probabilities and Derived Strengths of Preferences", Internal report $87 \mathrm{MA} \mathrm{03,} \mathrm{University} \mathrm{of} \mathrm{Nijmegen,} \mathrm{Department} \mathrm{of} \mathrm{Mathematical}$ Psychology, Nijmegen, The Netherlands.

Wakker, P.P.: 1988, 'The Algebraic Versus the Topological Approach to Additive Representations', Journal of Mathematical Psychology 32, 421-435.

Wakker, P.P.: 1989a, Additive Representations of Preferences, A New Foundation of Decision Analysis, Kluwer, Dordrecht.

Wakker, P.P.: 1989b, 'Transforming Probabilities without Violating Stochastic Dominance', In Roskam, E.E.Ch.I. (Ed.): 1989, Mathematical Psychology in Progress, Springer, Berlin, pp. 29-47.

Wakker, P.P.: 1990a, 'Under Stochastic Dominance Choquet-Expected Utility and Anticipated Utility are Identical', Theory and Decision 29, 119-132.

Wakker, P.P.: 1990b, 'Characterizing Optimism and Pessimism Directly through Comonotonicity', Journal of Economic Theory 52, 453-463.

Wakker, P.P.: 1991a, 'Additive Representations on Rank-Ordered Sets. I. The Algebraic Approach', Joumal of Mathematical Psychology 35, 501-531.

Wakker, P.P.: 1991b, 'Additive Representations of Preferences, A New Foundation of Decision Analysis; The Algebraic Approach', In Doignon, J.P. and Falmagne, J.C. (Eds.): 1991, Mathematical Psychology: Current Developments, Springer, Berlin, pp. 71-87.

Wakker, P.P.: 1993a, 'Additive Representations on Rank-Ordered Sets. II. The Topological Approach', Journal of Mathematical Economics 22, 1-26.

Wakker, P.P.: 1993b, 'Counterexamples to Segal's Measure Representation Theorem', Journal of Risk and Uncertainty 6, 91-98.

Wakker, P.P.: 1993c, 'Unbounded Utility for Savage's 'Foundations of Statistics', and other Models', Mathematics of Operations Research 18, 446-485.

Wakker, P.P.: 1993d, 'Savage's Axioms Usually Imply Violation of Strict Stochastic Dominance', The Review of Economic Studies 60, 487-493.

Wakker, P.P. and Tversky, A.: 1991, 'An Axiomatization of Cumulative Prospect Theory', Journal of Risk and Uncertainty, forthcoming.

Yaari, M.E.: 1977, 'A Note on Separability and Quasi-Concavity', Econometrica 45, $1183-1186$. 
Yaari, M.E.: 1987a, 'The Dual Theory of Choice under Risk', Econometrica 55, 95-115. Yaari, M.E.: 1987b, 'Univariate and Multivatiate Comparisons of Risk Aversion: a New Approach', In Heller, W.P., Starr, R.M. and Starrett, D.A. (Eds.): 1987, Uncertainty, Information and Communication: Essays in Honor of Kenneth J. Arrow, Cambridge University Press, Cambridge, Vol. III, pp. 173-187. 